\title{
Remarks on Numerical Experiments of the Allen-Cahn Equations with Constraint via Yosida Approximation
}

\author{
Tomoyuki Suzuki, ${ }^{1}$ Keisuke Takasao, ${ }^{2}$ and Noriaki Yamazaki ${ }^{1}$ \\ ${ }^{1}$ Department of Mathematics, Faculty of Engineering, Kanagawa University, 3-27-1 Rokkakubashi, Kanagawa-ku, \\ Yokohama 221-8686, Japan \\ ${ }^{2}$ Graduate School of Mathematical Sciences, University of Tokyo, 3-8-1 Komaba, Meguro-ku, Tokyo 153-8914, Japan
}

Correspondence should be addressed to Noriaki Yamazaki; noriaki@kanagawa-u.ac.jp

Received 16 February 2016; Accepted 5 April 2016

Academic Editor: Yinnian He

Copyright (C) 2016 Tomoyuki Suzuki et al. This is an open access article distributed under the Creative Commons Attribution License, which permits unrestricted use, distribution, and reproduction in any medium, provided the original work is properly cited.

\begin{abstract}
We consider a one-dimensional Allen-Cahn equation with constraint from the viewpoint of numerical analysis. The constraint is provided by the subdifferential of the indicator function on the closed interval, which is the multivalued function. Therefore, it is very difficult to perform a numerical experiment of our equation. In this paper we approximate the constraint by the Yosida approximation. Then, we study the approximating system of the original model numerically. In particular, we give the criteria for the standard forward Euler method to give the stable numerical experiments of the approximating equation. Moreover, we provide the numerical experiments of the approximating equation.
\end{abstract}

\section{Introduction}

In this paper, for each $\varepsilon \in(0,1]$ we consider the following Allen-Cahn equation with constraint from the viewpoint of numerical analysis:

$$
\begin{aligned}
u_{t}^{\varepsilon}-u_{x x}^{\varepsilon}+\frac{\partial I_{[-1,1]}\left(u^{\varepsilon}\right)}{\varepsilon^{2}} & \ni \frac{u^{\varepsilon}}{\varepsilon^{2}} \quad \text { in } Q:=(0, T) \times(0,1), \\
u_{x}^{\varepsilon}(t, 0) & =u_{x}^{\varepsilon}(t, 1)=0, \quad t \in(0, T), \\
u^{\varepsilon}(0, x) & =u_{0}^{\varepsilon}(x), \quad x \in(0,1),
\end{aligned}
$$

where $0<T<+\infty$ and $u_{0}^{\varepsilon}$ is given initial data. Also, the constraint $\partial I_{[-1,1]}(\cdot)$ is the subdifferential of the indicator function $I_{[-1,1]}(\cdot)$ on the closed interval $[-1,1]$ defined by

$$
I_{[-1,1]}(z):= \begin{cases}0, & \text { if } z \in[-1,1] \\ +\infty, & \text { otherwise. }\end{cases}
$$

More precisely, $\partial I_{[-1,1]}(\cdot)$ is a set-valued mapping defined by

$$
\partial I_{[-1,1]}(z):= \begin{cases}\emptyset, & \text { if } z<-1 \text { or } z>1, \\ {[0, \infty),} & \text { if } z=1, \\ \{0\}, & \text { if }-1<z<1, \\ (-\infty, 0], & \text { if } z=-1 .\end{cases}
$$

The Allen-Cahn equation was proposed to describe the macroscopic motion of phase boundaries. In the physical context, the function $u^{\varepsilon}=u^{\varepsilon}(t, x)$ in $(P)^{\varepsilon}:=\{(1),(2),(3)\}$ is the nonconserved order parameter that characterizes the physical structure. For instance, let $v=v(t, x)$ be the local ratio of the volume of pure liquid relative to that of pure solid at time $t$ and position $x \in(0,1)$, defined by

$$
\begin{aligned}
& v(t, x) \\
& :=\lim _{r \downarrow 0} \frac{\text { the volume of pure liquid in } B_{r}(x) \text { at time } t}{\left|B_{r}(x)\right|},
\end{aligned}
$$

where $B_{r}(x)$ is the ball in $\mathbb{R}$ with center $x$ and radius $r$ and $\left|B_{r}(x)\right|$ denotes its volume. Put $u^{\varepsilon}(t, x):=2 v(t, x)-1$ for any 
$(t, x) \in Q$. Then, we observe that $u^{\varepsilon}(t, x)$ is the nonconserved order parameter that characterizes the physical structure:

$$
\begin{aligned}
u^{\varepsilon}(t, x) & =1 \quad \text { on the pure liquid region, } \\
u^{\varepsilon}(t, x) & =-1 \quad \text { on the pure solid region, } \\
-1 & <u^{\varepsilon}(t, x)<1 \quad \text { on the mixture region. }
\end{aligned}
$$

There is a vast amount of literature on the Allen-Cahn equation with or without constraint $\partial I_{[-1,1]}(\cdot)$. For these studies, we refer to [1-16]. In particular, Chen and Elliott [5] considered the singular limit of $(P)^{\varepsilon}$ as $\varepsilon \rightarrow 0$ in the general bounded domain $\Omega\left(\subset \mathbb{R}^{N}\right.$ with $\left.N \geq 1\right)$.

Also, there is a vast amount of literature on the numerical analysis of the Allen-Cahn equation without constraint $\partial I_{[-1,1]}(\cdot)$. For these studies, we refer to [17-21].

Note that the constraint $\partial I_{[-1,1]}(\cdot)$ is the multivalued function. Therefore, it is very difficult to apply the numerical methods developed by [17-21] to $(P)^{\varepsilon}$. Hence, it is difficult to perform a numerical experiment of $(P)^{\varepsilon}$. Recently, Blank et al. [2] proposed as a numerical method a primal-dual active set algorithm for the local and nonlocal Allen-Cahn variational inequalities with constraint. Also, Farshbaf-Shaker et al. [8] gave the results of the limit of a solution $u^{\varepsilon}$ and an element of $\partial I_{[-1,1]}\left(u^{\varepsilon}\right)$, called the Lagrange multiplier, to $(P)^{\varepsilon}$ as $\varepsilon \rightarrow 0$. Moreover, they [9] gave the numerical experiment to $(P)^{\varepsilon}$ via the Lagrange multiplier in one dimension of space for sufficient small $\varepsilon \in(0,1]$. Furthermore, they [9] considered the approximating method, called the Yosida approximation. More precisely, for $\delta>0$, the Yosida approximation $\left(\partial I_{[-1,1]}\right)_{\delta}(\cdot)$ of $\partial I_{[-1,1]}(\cdot)$ is defined by

$$
\left(\partial I_{[-1,1]}\right)_{\delta}(z)=\frac{[z-1]^{+}-[-1-z]^{+}}{\delta}, \quad \forall z \in \mathbb{R},
$$

where $[z]^{+}$is the positive part of $z$. Then, for each $\delta>0$, they [9] considered the following approximation problem of $(P)^{\varepsilon}$ :

$$
(P)_{\delta}^{\varepsilon} \begin{cases}\left(u_{\delta}^{\varepsilon}\right)_{t}-\left(u_{\delta}^{\varepsilon}\right)_{x x}+\frac{\left(\partial I_{[-1,1]}\right)_{\delta}\left(u_{\delta}^{\varepsilon}\right)}{\varepsilon^{2}}=\frac{u_{\delta}^{\varepsilon}}{\varepsilon^{2}} & \text { in } Q:=(0, T) \times(0,1) \\ \left(u_{\delta}^{\varepsilon}\right)_{x}(t, 0)=\left(u_{\delta}^{\varepsilon}\right)_{x}(t, 1)=0, & t \in(0, T) \\ u_{\delta}^{\varepsilon}(0, x)=u_{0}^{\varepsilon}(x), & x \in(0,1) .\end{cases}
$$

In [9, Remark 4.2], Figure 1 shows the unstable numerical result to $(P)_{\delta}^{\varepsilon}$ was given by the standard explicit finite difference scheme to $(P)_{\delta}^{\varepsilon}$.

From Figure 1, we observe that we have to choose the suitable constants $\varepsilon$ and $\delta$ and the mesh size of time $\Delta t$ and space $\Delta x$ in order to get the stable numerical results of $(P)_{\delta}^{\varepsilon}$. Therefore, in this paper, for each $\varepsilon>0$ and $\delta>0$, we give the criteria for the standard explicit finite difference scheme to provide the stable numerical experiments of $(P)_{\delta}^{\varepsilon}$. To this end, we first consider the following ODE problem, denoted by $(E)_{\delta}^{\varepsilon}$ :

$$
(E)_{\delta}^{\varepsilon} \begin{cases}\left(u_{\delta}^{\varepsilon}\right)_{t}+\frac{\left(\partial I_{[-1,1]}\right)_{\delta}\left(u_{\delta}^{\varepsilon}\right)}{\varepsilon^{2}}=\frac{u_{\delta}^{\varepsilon}}{\varepsilon^{2}} & \text { in } \mathbb{R}, \text { for } t \in(0, T), \\ u_{\delta}^{\varepsilon}(0)=u_{0}^{\varepsilon} & \text { in } \mathbb{R} .\end{cases}
$$

Then, we give the criteria to get the stable numerical experiments of $(E)_{\delta}^{\varepsilon}$. Also, we give some numerical experiments of $(E)_{\delta}^{\varepsilon}$. Moreover, we show the criteria to get the stable numerical experiments of PDE problem $(P)_{\delta}^{\varepsilon}$. Therefore, the main novelties are the following:

(a) We give the criteria to get the stable numerical experiments of the ODE problem $(E)_{\delta}^{\varepsilon}$. Also, we provide the numerical experiments to $(E)_{\delta}^{\varepsilon}$ for sufficient small $\varepsilon \in(0,1]$ and $\delta \in(0,1]$.

(b) We give the criteria to get the stable numerical experiments of the PDE problem $(P)_{\delta}^{\varepsilon}$. Also, we provide the numerical experiments to $(P)_{\delta}^{\varepsilon}$ for sufficient small $\varepsilon \in(0,1]$ and $\delta \in(0,1]$.

The plan of this paper is as follows. In Section 2, we mention the solvability and convergence result of $(E)_{\delta}^{\varepsilon}$. In Section 3, we consider $(E)_{\delta}^{\varepsilon}$ numerically. Then, we prove the main result (Theorem 7 ) corresponding to item (a) listed above. Also, we provide the numerical experiments to $(E)_{\delta}^{\varepsilon}$ for sufficient small $\varepsilon \in(0,1]$ and $\delta \in(0,1]$. In Section 4 , we mention the solvability and convergence result of $(P)_{\delta}^{\varepsilon}$. In the final Section 5, we consider $(P)_{\delta}^{\varepsilon}$ from the viewpoint of numerical analysis. Then, we prove the main result (Theorem 16) corresponding to item (b) listed above. Also, we provide the numerical experiments to $(P)_{\delta}^{\varepsilon}$ for sufficient small $\varepsilon \in(0,1]$ and $\delta \in(0,1]$.

Notations and Basic Assumptions. Throughout this paper, we put $H:=L^{2}(0,1)$ with the usual real Hilbert space structure. The inner product and norm in $H$ are denoted by $(\cdot, \cdot)_{H}$ and by $|\cdot|_{H}$, respectively. We also put $V:=H^{1}(0,1)$ with the usual $\operatorname{norm}|z|_{V}:=\left\{|z|_{H}^{2}+\left|z_{x}\right|_{H}^{2}\right\}^{1 / 2}$ for $z \in V$.

In Sections 2 and 4, we use some techniques of proper (i.e., not identically equal to infinity), l.s.c. (lower semicontinuous), and convex functions and their subdifferentials, which are useful in the systematic study of variational inequalities. So, let us outline some notations and definitions. Let $W$ be the real Hilbert space with the inner product $(\cdot, \cdot)_{W}$. For a proper, l.s.c., and convex function $\psi: W \rightarrow \mathbb{R} \cup\{+\infty\}$, the effective domain $D(\psi)$ is defined by

$$
D(\psi):=\{z \in W ; \psi(z)<\infty\} .
$$




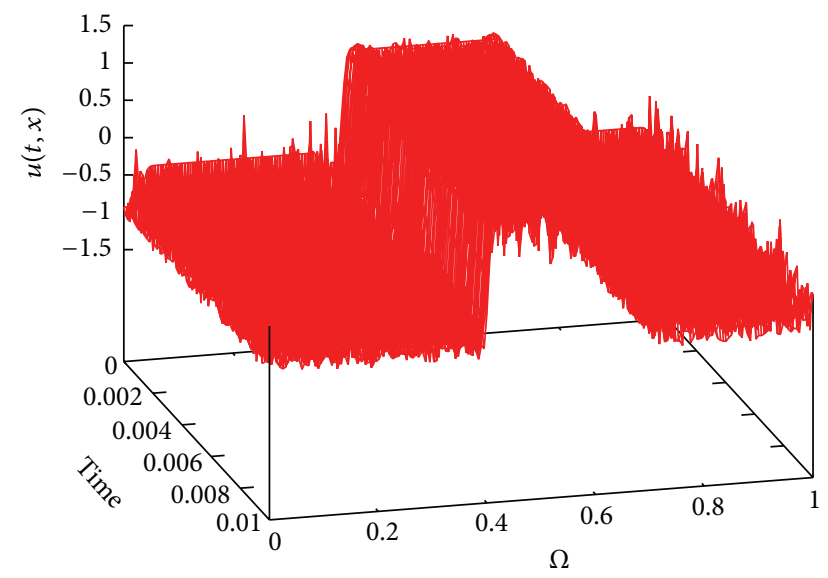

FIGURE 1: Behavior of a solution to $(P)_{\delta}^{\varepsilon}$ with $\varepsilon=0.007$ and $\delta=0.01$.

The subdifferential of $\psi$ is a possibly multivalued operator in $W$ and is defined by $z^{*} \in \partial \psi(z)$ if and only if

$$
\begin{gathered}
z \in D(\psi) \\
\left(z^{*}, y-z\right)_{W} \leq \psi(y)-\psi(z) \quad \forall y \in W .
\end{gathered}
$$

For various properties and related notions of the proper, l.s.c., and convex function $\psi$ and its subdifferential $\partial \psi$, we refer to the monograph by Brézis [22].

\section{Solvability and Convergence Results of $(E)_{\delta}^{\varepsilon}$}

We begin by giving the rigorous definition of solutions to our problem $(E)_{\delta}^{\varepsilon}(\varepsilon \in(0,1]$ and $\delta \in(0,1])$.
Definition 1. Let $\varepsilon \in(0,1], \delta \in(0,1]$, and $u_{0}^{\varepsilon} \in \mathbb{R}$. Then, a function $u_{\delta}^{\varepsilon}:[0, T] \rightarrow \mathbb{R}$ is called a solution to $(E)_{\delta}^{\varepsilon}$ on $[0, T]$, if the following conditions are satisfied:

(i) $u_{\delta}^{\varepsilon} \in W^{1,2}(0, T)$.

(ii) The following equation holds:

$\left(u_{\delta}^{\varepsilon}\right)_{t}+\frac{\left(\partial I_{[-1,1]}\right)_{\delta}\left(u_{\delta}^{\varepsilon}\right)}{\varepsilon^{2}}=\frac{u_{\delta}^{\varepsilon}}{\varepsilon^{2}} \quad$ in $\mathbb{R}$, for $t \in(0, T)$.

(iii) $u_{\delta}^{\varepsilon}(0)=u_{0}^{\varepsilon}$ in $\mathbb{R}$

Now, we show the solvability result of $(E)_{\delta}^{\varepsilon}$ on $[0, T]$.

Proposition 2. Let $\varepsilon \in(0,1], \delta \in(0,1]$, and $u_{0}^{\varepsilon} \in \mathbb{R}$ with $\left|u_{0}^{\varepsilon}\right| \leq 1$. Then, there exists a unique solution $u_{\delta}^{\varepsilon}$ to $(E)_{\delta}^{\varepsilon}$ on $[0, T]$ in the sense of Definition 1.

Proof. We can prove the uniqueness of solutions to $(E)_{\delta}^{\varepsilon}$ on $[0, T]$ by the quite standard arguments: monotonicity and Gronwall's inequality.

Also, we can show the existence of solutions to $(E)_{\delta}^{\varepsilon}$ on $[0, T]$ applying the abstract theory of evolution equations governed by subdifferentials. Indeed, we define a function $\left(I_{[-1,1]}\right)_{\delta}(\cdot)$ on $\mathbb{R}$ by

$$
\left(I_{[-1,1]}\right)_{\delta}(z)=\frac{\left|[z-1]^{+}\right|^{2}+\left|[-1-z]^{+}\right|^{2}}{2 \delta}, \quad \forall z \in \mathbb{R}
$$

Clearly, $\left(I_{[-1,1]}\right)_{\delta}(\cdot)$ is proper, l.s.c., and convex on $\mathbb{R}$ with $\partial\left(I_{[-1,1]}\right)_{\delta}(\cdot)=\left(\partial I_{[-1,1]}\right)_{\delta}(\cdot)$ in $\mathbb{R}$, where $\left(\partial I_{[-1,1]}\right)_{\delta}(\cdot)$ is the Yosida approximation of $\partial I_{[-1,1]}(\cdot)$ defined by $(8)$.

Note that the problem $(E)_{\delta}^{\varepsilon}$ can be rewritten as in an abstract framework of the form

$$
(E P)_{\delta}^{\varepsilon} \begin{cases}\frac{d}{d t} u_{\delta}^{\varepsilon}(t)+\frac{1}{\varepsilon^{2}} \partial\left(I_{[-1,1]}\right)_{\delta}\left(u_{\delta}^{\varepsilon}(t)\right)-\frac{1}{\varepsilon^{2}} u_{\delta}^{\varepsilon}(t)=0 & \text { in } \mathbb{R}, \text { for } t \in(0, T), \\ u_{\delta}^{\varepsilon}(0)=u_{0}^{\varepsilon} & \text { in } \mathbb{R} .\end{cases}
$$

Therefore, applying the Lipschitz perturbation theory of abstract evolution equations (cf. [23-25]), we can show the existence of a solution $u_{\delta}^{\varepsilon}$ to $(E P)_{\delta}^{\varepsilon}$, hence, $(E)_{\delta}^{\varepsilon}$, on $[0, T]$ for each $\varepsilon \in(0,1]$ and $\delta \in(0,1]$ in the sense of Definition 1 . Thus, the proof of Proposition 2 has been completed.

Next, we show the convergence result of $(E)_{\delta}^{\varepsilon}$ as $\delta \rightarrow 0$. To this end, we recall a notion of convergence for convex functions, developed by Mosco [26].

Definition 3 (cf. [26]). Let $\psi$ and $\psi_{n}(n \in \mathbb{N})$ be proper, l.s.c., and convex functions on a Hilbert space $W$. Then, one says that $\psi_{n}$ converges to $\psi$ on $W$ in the sense of Mosco [26] as $n \rightarrow \infty$, if the following two conditions are satisfied: (i) for any subsequence $\left\{\psi_{n_{k}}\right\} \subset\left\{\psi_{n}\right\}$, if $z_{k} \rightarrow z$ weakly in $W$ as $k \rightarrow \infty$, then

$$
\liminf _{k \rightarrow \infty} \psi_{n_{k}}\left(z_{k}\right) \geq \psi(z)
$$

(ii) for any $z \in D(\psi)$, there is a sequence $\left\{z_{n}\right\}$ in $W$ such that

$$
\begin{aligned}
z_{n} & \longrightarrow z \text { in } W \text { as } n \longrightarrow \infty, \\
\lim _{n \rightarrow \infty} \psi_{n}\left(z_{n}\right) & =\psi(z) .
\end{aligned}
$$

It is well known that the following lemma holds. Therefore, we omit the detailed proof. 
Lemma 4 (cf. [27, Section 5], [22, Chapter 2], and [28, Section 2]). Consider

$$
\left(I_{[-1,1]}\right)_{\delta}(\cdot) \longrightarrow I_{[-1,1]}(\cdot)
$$

on $\mathbb{R}$ in the sense of Mosco [26] as $\delta \longrightarrow 0$.

By Lemma 4 and the general convergence theory of evolution equations, we get the following result. We omit the detailed proof.

Proposition 5 (cf. [27, Section 5], [28, Section 2]). Let $\varepsilon \in$ $(0,1], \delta \in(0,1]$, and $u_{0}^{\varepsilon} \in \mathbb{R}$ with $\left|u_{0}^{\varepsilon}\right| \leq 1$. Also, let $u_{\delta}^{\varepsilon}$ be the unique solution to $(E)_{\delta}^{\varepsilon}$ on $[0, T]$. Then, there exists a unique function $u^{\varepsilon} \in W^{1,2}(0, T)$ such that

$$
u_{\delta}^{\varepsilon} \longrightarrow u^{\varepsilon} \quad \text { in } C([0, T]) \text { as } \delta \longrightarrow 0
$$

and $u^{\varepsilon}$ is the unique solution of the following problem $(E)^{\varepsilon}$ on $[0, T]$ :

$$
(E)^{\varepsilon} \begin{cases}u_{t}^{\varepsilon}+\frac{\partial I_{[-1,1]}\left(u^{\varepsilon}\right)}{\varepsilon^{2}} \ni \frac{u^{\varepsilon}}{\varepsilon^{2}} & \text { in } \mathbb{R}, \text { for } t \in(0, T), \\ u^{\varepsilon}(0)=u_{0}^{\varepsilon} & \text { in } \mathbb{R} .\end{cases}
$$

\section{Stable Criteria and Numerical Experiments for $(E)_{\delta}^{\varepsilon}$}

In this section we consider $(E)_{\delta}^{\varepsilon}$ from the viewpoint of numerical analysis.

Remark 6. Note from Proposition 5 that $(E)_{\delta}^{\varepsilon}$ is the approximating problem of $(E)^{\varepsilon}$. Also note from (5) that the constraint $\partial I_{[-1,1]}(\cdot)$ is the multivalued function. Therefore, it is very difficult to study $(E)^{\varepsilon}$ numerically.

In order to perform the numerical experiments of $(E)_{\delta}^{\varepsilon}$ via the standard forward Euler method, we consider the following explicit finite difference scheme to $(E)_{\delta}^{\varepsilon}$, denoted by $(D E)_{\delta}^{\varepsilon}$ :

$$
(D E)_{\delta}^{\varepsilon} \begin{cases}\frac{u^{n+1}-u^{n}}{\Delta t}+\frac{\left(\partial I_{[-1,1]}\right)_{\delta}\left(u^{n}\right)}{\varepsilon^{2}}=\frac{u^{n}}{\varepsilon^{2}} & \text { in } \mathbb{R}, \text { for } n=0,1,2, \ldots, N_{t}, \\ u^{0}=u_{0}^{\varepsilon} & \text { in } \mathbb{R},\end{cases}
$$

where $\Delta t$ is the mesh size of time and $N_{t}$ is the integer part of number $T / \Delta t$.

We observe that $u^{n}$ is the approximating solution of $(E)_{\delta}^{\varepsilon}$ at the time $t=n \Delta t$. Also, we observe that the explicit finite difference scheme $(D E)_{\delta}^{\varepsilon}$ converges to $(E)_{\delta}^{\varepsilon}$ as $\Delta t \rightarrow 0$ since $(D E)_{\delta}^{\varepsilon}$ is the standard time discretization scheme for $(E)_{\delta}^{\varepsilon}$.

In Figure 2, we give the unstable numerical experiment of $(D E)_{\delta}^{\varepsilon}$ in the case when $T=0.002, \varepsilon=0.003, \delta=0.01$, the initial data $u_{0}^{\varepsilon}=0.1$, and the mesh size of time $\Delta t=0.000001$.

From Figure 2, we observe that we have to choose the suitable constants $\varepsilon$ and $\delta$ and the mesh size of time $\Delta t$ in order to get the stable numerical results of $(D E)_{\delta}^{\varepsilon}$.

Now, let us mention the first main result in this paper, which is concerned with the criteria to give the stable numerical experiments of $(D E)_{\delta}^{\varepsilon}$.

Theorem 7. Let $\varepsilon \in(0,1], \delta \in(0,1)$, and $\Delta t \in(0,1]$. Assume $u_{0}^{\varepsilon} \in(0,1]$ (resp., $u_{0}^{\varepsilon} \in[-1,0)$ ) and $T=\infty$. Let $\left\{u^{n} ; n \geq 0\right\}$ be the solution to $(D E)_{\delta}^{\varepsilon}$. Then, one has the following:

(i) If $\Delta t \in\left(0, \delta \varepsilon^{2} /(1-\delta)\right)$, $u^{n}$ converges to $1 /(1-\delta)$ (resp., $-1 /(1-\delta))$ monotonically as $n \rightarrow \infty$.

(ii) If $\Delta t \in\left(\delta \varepsilon^{2} /(1-\delta), 2 \delta \varepsilon^{2} /(1-\delta)\right), u^{n}$ oscillates and converges to $1 /(1-\delta)$ (resp., $-1 /(1-\delta))$ as $n \rightarrow \infty$.

Proof. We give the proof of Theorem 7 in the case of the initial value $u_{0}^{\varepsilon} \in(0,1]$.
For simplicity, we set

$$
f_{\delta}(z):=\left(\partial I_{[-1,1]}\right)_{\delta}(z)-z \quad \text { for } z \in \mathbb{R} .
$$

Then, we observe that

$$
f_{\delta}(z)= \begin{cases}\frac{1+z}{\delta}-z, & \text { if } z \leq-1, \\ -z, & \text { if } z \in[-1,1], \\ \frac{z-1}{\delta}-z, & \text { if } z \geq 1,\end{cases}
$$

and $z=0,1 /(1-\delta),-1 /(1-\delta)$ are the zero points of $f_{\delta}(\cdot)$. Also, we observe that the difference equation $(D E)_{\delta}^{\varepsilon}$ is reformulated in the following form:

$$
u^{n+1}=u^{n}-\frac{\Delta t}{\varepsilon^{2}} f_{\delta}\left(u^{n}\right) \quad \text { in } \mathbb{R}, \text { for } n=0,1,2, \ldots
$$

Note from (23) and (24) that if $u^{n} \in(0,1]$ we have

$$
u^{n+1}=u^{n}-\frac{\Delta t}{\varepsilon^{2}} f_{\delta}\left(u^{n}\right)=u^{n}-\frac{\Delta t}{\varepsilon^{2}} \cdot\left(-u^{n}\right) \geq u^{n},
$$

which implies that $u^{n}$ is increasing with respect to $n$ until $u^{n+1} \geq 1$.

Now, we prove (i). To this end, we assume that $\Delta t \epsilon$ $\left(0, \delta \varepsilon^{2} /(1-\delta)\right)$. At first, by the mathematical induction, we show

$$
u^{i} \in\left(0, \frac{1}{1-\delta}\right) \quad \forall i \geq 0
$$




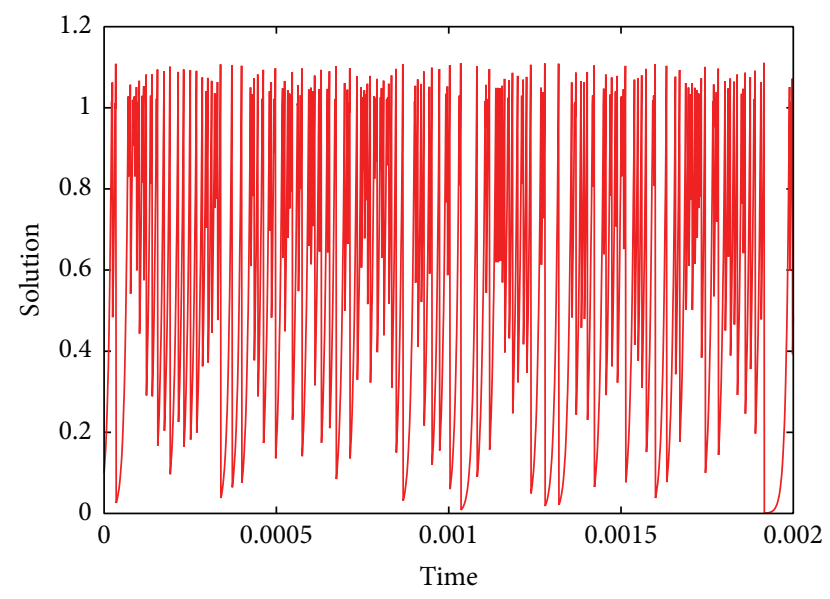

FIGURE 2: Behavior of a solution $u^{n}$ to $(D E)_{\delta}^{\varepsilon}$ with $\varepsilon=0.003$ and $\delta=0.01$.

Clearly (26) holds for $i=0$ because of $u^{0}=u_{0}^{\varepsilon} \in(0,1]$.

Now, we assume that (26) holds for all $i=0,1, \ldots, n$. Suppose $u^{n} \in(0,1]$. Then, we infer from (25) that

$$
u^{n+1}=\left(1+\frac{\Delta t}{\varepsilon^{2}}\right) u^{n} \leq 1+\frac{\Delta t}{\varepsilon^{2}}<1+\frac{\delta}{1-\delta}=\frac{1}{1-\delta} .
$$

Therefore, by (25) and the inequality as above, we observe that

$$
u^{n+1} \in\left(0, \frac{1}{1-\delta}\right), \quad \text { if } u^{n} \in(0,1]
$$

Next, if $u^{n} \in[1,1 /(1-\delta))$, we observe from (23) and (24) that

$$
\begin{aligned}
u^{n} & \leq u^{n+1}=u^{n}-\frac{\Delta t}{\varepsilon^{2}} f_{\delta}\left(u^{n}\right) \\
& =u^{n}-\frac{\Delta t}{\varepsilon^{2}} \cdot\left(\frac{u^{n}-1}{\delta}-u^{n}\right) \\
& =u^{n}+\frac{\Delta t}{\varepsilon^{2}} \cdot \frac{1-(1-\delta) u^{n}}{\delta}<u^{n}+\frac{1-(1-\delta) u^{n}}{1-\delta} \\
& =\frac{1}{1-\delta},
\end{aligned}
$$

which implies that

$$
u^{n+1} \in\left[1, \frac{1}{1-\delta}\right), \quad \text { if } u^{n} \in\left[1, \frac{1}{1-\delta}\right) .
$$

From (28) and (30) we infer that (26) holds for $i=n+1$. Therefore, we conclude from the mathematical induction that (26) holds.

Also, by (23) and (26) we observe that $f_{\delta}\left(u^{n}\right) \leq 0$ for all $n \geq 0$. Therefore, we observe from (24) that

$$
u^{n+1}=u^{n}-\frac{\Delta t}{\varepsilon^{2}} f_{\delta}\left(u^{n}\right) \geq u^{n} \quad \forall n \geq 0 .
$$

Therefore, we infer from (26) and (31) that $\left\{u^{n} ; n \geq 0\right\}$ is a bounded and increasing sequence with respect to $n$. Thus, there exists a point $u^{\infty} \in \mathbb{R}$ such that

$$
u^{n} \longrightarrow u^{\infty} \text { in } \mathbb{R} \text { as } n \longrightarrow \infty \text {. }
$$

Taking the limit in (24), we observe from the continuity of $f_{\delta}(\cdot)$ that $u^{\infty}=1 /(1-\delta)$, which is the zero point of $f_{\delta}(\cdot)$. Hence, the proof of (i) has been completed.

Next, we show (ii). To this end, we assume that $\Delta t \in$ $\left(\delta \varepsilon^{2} /(1-\delta), 2 \delta \varepsilon^{2} /(1-\delta)\right)$. Then, we can find the minimal number $n_{0} \in \mathbb{N}$ so that

$$
\begin{aligned}
& u^{n_{0}} \in\left(1, \frac{1+\delta}{1-\delta}\right), \\
& u^{i} \in(0,1] \quad \forall i=0,1, \ldots, n_{0}-1 .
\end{aligned}
$$

Indeed, if $u^{i} \in(0,1]$ for all $i=0,1, \ldots, k$, we observe from (25) that

$$
\begin{aligned}
u^{k+1} & =u^{k}-\frac{\Delta t}{\varepsilon^{2}} f_{\delta}\left(u^{k}\right)=\left(1+\frac{\Delta t}{\varepsilon^{2}}\right) u^{k} \\
& =\left(1+\frac{\Delta t}{\varepsilon^{2}}\right)^{2} u^{k-1}=\cdots=\left(1+\frac{\Delta t}{\varepsilon^{2}}\right)^{k+1} u^{0} .
\end{aligned}
$$

Taking into account (34), $u_{0} \in(0,1]$, and

$$
1+\frac{\Delta t}{\varepsilon^{2}}>1+\frac{\delta}{1-\delta}>1,
$$

we can find the minimal number $n_{0} \in \mathbb{N}$ so that

$$
\begin{aligned}
& u^{n_{0}}>1, \\
& u^{i} \in(0,1] \quad \forall i=0,1, \ldots, n_{0}-1 .
\end{aligned}
$$

Also, we observe from (25) that

$$
\begin{aligned}
u^{n_{0}} & =u^{n_{0}-1}-\frac{\Delta t}{\varepsilon^{2}} f_{\delta}\left(u^{n_{0}-1}\right)=\left(1+\frac{\Delta t}{\varepsilon^{2}}\right) u^{n_{0}-1} \\
& <\left(1+\frac{2 \delta}{1-\delta}\right) \cdot 1=\frac{1+\delta}{1-\delta},
\end{aligned}
$$

and thus, (33) holds.

To show (ii), we put

$$
\Delta t:=\frac{\delta \varepsilon^{2}}{1-\delta} \tau \quad \text { for some } \tau \in(1,2)
$$

Then, we observe from (23) and (24) that

$$
\begin{aligned}
u^{n_{0}+1} & =u^{n_{0}}-\frac{\Delta t}{\varepsilon^{2}} f_{\delta}\left(u^{n_{0}}\right)=u^{n_{0}}+\frac{\Delta t}{\varepsilon^{2}} \cdot \frac{1-(1-\delta) u^{n_{0}}}{\delta} \\
& =(1-\tau) u^{n_{0}}+\frac{\tau}{1-\delta} .
\end{aligned}
$$

From (39) it follows that

$$
\frac{u^{n_{0}+1}+(\tau-1) u^{n_{0}}}{\tau}=\frac{1}{1-\delta} .
$$


Therefore, we observe from $(40)$ and $\tau \in(1,2)$ that the zero point $1 /(1-\delta)$ of $f_{\delta}(\cdot)$ is in the interval between $u^{n_{0}}$ and $u^{n_{0}+1}$.

Also, by (39) we observe that

$$
\begin{aligned}
u^{n_{0}+1} & =(1-\tau) u^{n_{0}}+\frac{\tau}{1-\delta}>(1-\tau) \frac{1+\delta}{1-\delta}+\frac{\tau}{1-\delta} \\
& =\frac{1+\delta-\tau \delta}{1-\delta} \geq 1, \\
u^{n_{0}+1} & =(1-\tau) u^{n_{0}}+\frac{\tau}{1-\delta}<(1-\tau) \cdot 1+\frac{\tau}{1-\delta} \\
& =\frac{1-\delta+\tau \delta}{1-\delta} \leq \frac{1+\delta}{1-\delta},
\end{aligned}
$$

which implies that

$$
u^{n_{0}+1} \in\left(1, \frac{1+\delta}{1-\delta}\right)
$$

Therefore, by (33) and (40) and by repeating the procedure as above, we observe that

$$
u^{n} \in\left(1, \frac{1+\delta}{1-\delta}\right) \quad \forall n \geq n_{0}
$$

and $u^{n}$ oscillates around the zero point $1 /(1-\delta)$ for all $n \geq n_{0}$.

Also, we observe from (39) and (43) that

$$
\left|u^{n+1}-\frac{1}{1-\delta}\right|=|1-\tau|\left|u^{n}-\frac{1}{1-\delta}\right| \quad \forall n \geq n_{0} .
$$

Therefore, by $\tau \in(1,2)$, (43), and (44), there is a subsequence $\left\{n_{k}\right\}$ of $\{n\}$ such that $u^{n_{k}}$ oscillates and converges to $1 /(1-\delta)$ as $k \rightarrow \infty$. Hence, taking into account the uniqueness of the limit point, the proof of (ii) has been completed.

Remark 8. Assume $\Delta t \in\left[2 \delta \varepsilon^{2} /(1-\delta), \infty\right)$ and put $\Delta t:=$ $\delta \varepsilon^{2} \tau /(1-\delta)$ for some $\tau \geq 2$. Then, we observe that

$$
\begin{aligned}
& 1+\frac{\Delta t}{\varepsilon^{2}}>1+\frac{2 \delta}{1-\delta}>1, \\
& |1-\tau| \geq 1
\end{aligned}
$$

Therefore, we infer from the proof of Theorem 7 (cf. (34), (40), and (44)) that the solution $u^{n}$ to $(D E)_{\delta}^{\varepsilon}$ oscillates as $n \rightarrow \infty$, in general.

Remark 9. By (24) we easily observe that

$$
\begin{aligned}
& u^{n} \equiv 0 \quad \forall n \geq 1, \text { if } u_{0}=0, \\
& u^{n} \equiv \frac{1}{1-\delta} \quad \forall n \geq 1, \text { if } u_{0}=\frac{1}{1-\delta}, \\
& u^{n} \equiv \frac{-1}{1-\delta} \quad \forall n \geq 1, \text { if } u_{0}=\frac{-1}{1-\delta} .
\end{aligned}
$$

From (ii) of Theorem 7, we observe that $u_{n}$ oscillates and converges to the zero point of $f_{\delta}(\cdot)$ in the case when $\Delta t \in\left(\delta \varepsilon^{2} /(1-\delta), 2 \delta \varepsilon^{2} /(1-\delta)\right)$. However, in the case when $\Delta t=2 \delta \varepsilon^{2} /(1-\delta)$, we have the following special case that the solution to $(D E)_{\delta}^{\varepsilon}$ does not oscillate and coincides with the zero point of $f_{\delta}(\cdot)$ after some finite number of iterations.
Corollary 10. Let $\varepsilon \in(0,1], \delta \in(0,1), \Delta t=2 \delta \varepsilon^{2} /(1-\delta)$, and $n \in \mathbb{N}$. Assume $u_{0}^{\varepsilon}:=(1-\delta)^{n-1} /(1+\delta)^{n}$. Then, the solution to $(D E)_{\delta}^{\varepsilon}$ is given by

$$
u^{i}= \begin{cases}\frac{(1-\delta)^{n-1-i}}{(1+\delta)^{n-i}}, & \text { if } i=0,1, \ldots, n-1, \\ \frac{1}{1-\delta}, & \text { if } i \geq n .\end{cases}
$$

Proof. Note that $u_{0}^{\varepsilon}:=(1-\delta)^{n-1} /(1+\delta)^{n} \in(0,1)$. Therefore, by (23) and (24) we observe that

$$
\begin{aligned}
u^{1} & =u^{0}-\frac{\Delta t}{\varepsilon^{2}} f_{\delta}\left(u^{0}\right)=u_{0}^{\varepsilon}-\frac{2 \delta}{1-\delta}\left(-u_{0}^{\varepsilon}\right)=\frac{1+\delta}{1-\delta} u_{0}^{\varepsilon} \\
& =\frac{(1-\delta)^{n-2}}{(1+\delta)^{n-1}} .
\end{aligned}
$$

Similarly, we have

$$
u^{2}=u^{1}-\frac{\Delta t}{\varepsilon^{2}} f_{\delta}\left(u^{1}\right)=\frac{1+\delta}{1-\delta} u^{1}=\frac{(1-\delta)^{n-3}}{(1+\delta)^{n-2}} .
$$

Repeating this procedure, we observe that the solution to $(D E)_{\delta}^{\varepsilon}$ is given by (47).

Taking into account Theorem 7 , we provide the numerical experiments of $(D E)_{\delta}^{\varepsilon}$ as follows. To this end, we use the following numerical data.

\section{Numerical Data of $(D E)_{\delta}^{\varepsilon}$}

The numerical data are $T=0.002, \varepsilon=0.01, \delta=0.01$, and the initial data $u_{0}^{\varepsilon}=0.1$.

Then, we observe that

$$
\begin{aligned}
\frac{1}{1-\delta} & =\frac{1}{1-0.01}=1.010101010 \ldots, \\
\frac{\delta \varepsilon^{2}}{1-\delta} & =0.0000010101010 \ldots
\end{aligned}
$$

3.1. The Case When $\Delta t=0.000001$. Now we consider the case when $\Delta t=0.000001$. In this case, we have

$$
\frac{\delta \varepsilon^{2}}{1-\delta}=0.0000010101010 \cdots>\Delta t=0.000001,
$$

which implies that (i) of Theorem 7 holds. Thus, we have the stable numerical result of $(D E)_{\delta}^{\varepsilon}$. Indeed, we observe from Figure 3 and Table 1 that the solution to $(D E)_{\delta}^{\varepsilon}$ converges to the stationary solution $1 /(1-\delta)=1 /(1-0.01)=$ $1.010101010 \ldots$.

3.2. The Case When $\Delta t=0.000002$. Now we consider the case when $\Delta t=0.000002$. In this case, we have

$$
\begin{aligned}
\frac{\delta \varepsilon^{2}}{1-\delta} & =0.0000010101010 \cdots<\Delta t=0.000002 \\
& <\frac{2 \delta \varepsilon^{2}}{1-\delta},
\end{aligned}
$$


TABLE 1: Numerical data: $\Delta t=000001$.

\begin{tabular}{lc}
\hline Number of iterations $i$ & The value of $u^{i}$ \\
\hline 0 & 0.100000 \\
1 & 0.101000 \\
2 & 0.102010 \\
3 & 0.103030 \\
4 & 0.104060 \\
5 & 0.105101 \\
$\vdots$ & $\vdots$ \\
222 & 0.910636 \\
223 & 0.919743 \\
224 & 0.928940 \\
225 & 0.938230 \\
226 & 0.947612 \\
227 & 0.957088 \\
228 & 0.966659 \\
229 & 0.976325 \\
230 & 0.986089 \\
231 & 0.995950 \\
232 & 1.005909 \\
233 & 1.010059 \\
234 & 1.010101 \\
235 & 1.010101 \\
236 & 1.010101 \\
238 & 1.010101 \\
& 1.010101 \\
\hline
\end{tabular}

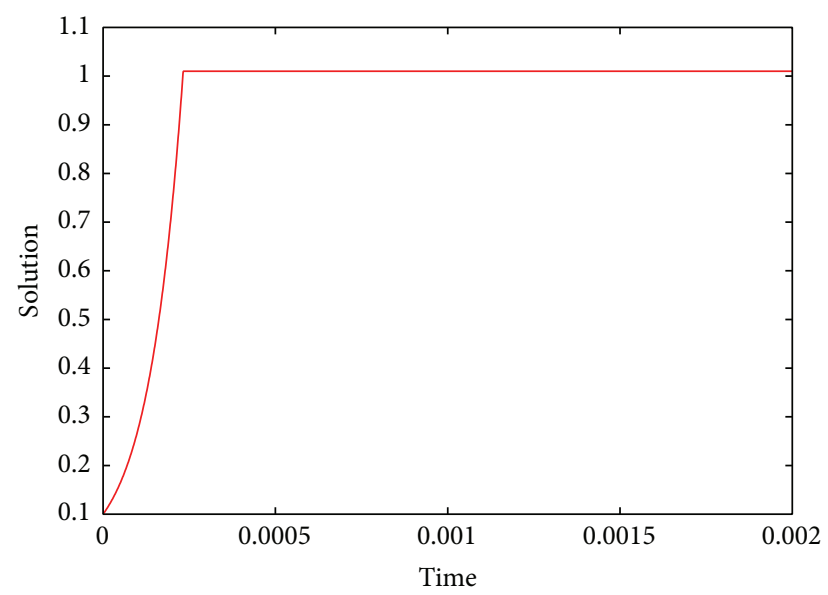

FIGURE 3: $\delta \varepsilon^{2} /(1-\delta)=0.0000010101010 \cdots>\Delta t=0.000001$.

which implies that (ii) of Theorem 7 holds. Thus, we observe from Figure 4 and Table 2 that the solution to $(\mathrm{DE})_{\delta}^{\varepsilon}$ oscillates and converges to the stationary solution $1 /(1-\delta)=1 /(1-$ $0.01)=1.010101010 \ldots$

3.3. The Case When $\Delta t=2 \delta \varepsilon^{2} /(1-\delta)$. Now we consider the case when $\Delta t=2 \delta \varepsilon^{2} /(1-\delta)=0.0000020202020 \ldots$. In this case, we observe Remark 8 . Indeed, we observe from Figure 5 and Table 3 that the solution to $(D E)_{\delta}^{\varepsilon}$ oscillates.
TABLE 2: Numerical data: $\Delta t=0.000002$.

\begin{tabular}{|c|c|}
\hline Number of iterations $i$ & The value of $u^{i}$ \\
\hline 0 & 0.100000 \\
\hline 1 & 0.102000 \\
\hline 2 & 0.104040 \\
\hline$\vdots$ & $\vdots$ \\
\hline 114 & 0.955916 \\
\hline 115 & 0.975034 \\
\hline 116 & 0.994535 \\
\hline 117 & 1.014425 \\
\hline 118 & 1.005863 \\
\hline 119 & 1.014254 \\
\hline 120 & 1.006031 \\
\hline 121 & 1.014090 \\
\hline 122 & 1.006192 \\
\hline 123 & 1.013932 \\
\hline 124 & 1.006347 \\
\hline 125 & 1.013780 \\
\hline 126 & 1.006496 \\
\hline 127 & 1.013634 \\
\hline 128 & 1.006638 \\
\hline 129 & 1.013494 \\
\hline$\vdots$ & $\vdots$ \\
\hline 560 & 1.010100 \\
\hline 561 & 1.010102 \\
\hline 562 & 1.010100 \\
\hline 563 & 1.010102 \\
\hline 564 & 1.010100 \\
\hline 565 & 1.010102 \\
\hline 566 & 1.010101 \\
\hline 567 & 1.010101 \\
\hline 568 & 1.010101 \\
\hline 569 & 1.010101 \\
\hline 570 & 1.010101 \\
\hline
\end{tabular}

3.4. The Case When $\Delta t=0.000005$. Now we consider the case when $\Delta t=0.000005$. In this case, we have

$$
2 \frac{\delta \varepsilon^{2}}{1-\delta}=0.0000020202020 \cdots<\Delta t=0.000005
$$

Therefore, we observe Remark 8. Indeed, we observe from Figure 6 that the solution to $(D E)_{\delta}^{\varepsilon}$ oscillates.

3.5. The Case When $\Delta t=15 \delta \varepsilon^{2} /(1-\delta)$. Now we consider the case when $\Delta t=15 \delta \varepsilon^{2} /(1-\delta)$. In this case, we observe Remark 8. Indeed, we observe from Figure 7 that the solution to $(D E)_{\delta}^{\varepsilon}$ oscillates between three zero points of $f_{\delta}(\cdot)$. 


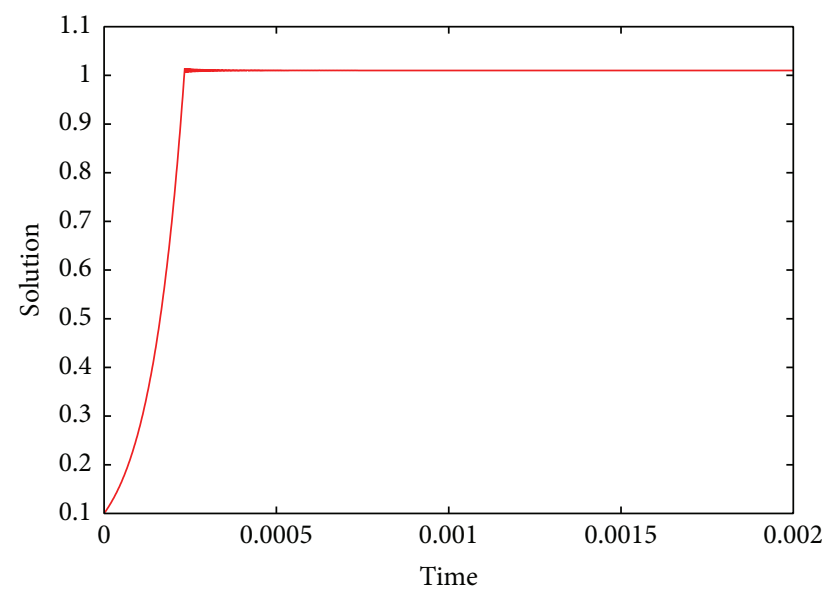

FIGURE 4: $\delta \varepsilon^{2} /(1-\delta)=0.0000010101010 \cdots<\Delta t=0.000002<$ $2 \delta \varepsilon^{2} /(1-\delta)$.

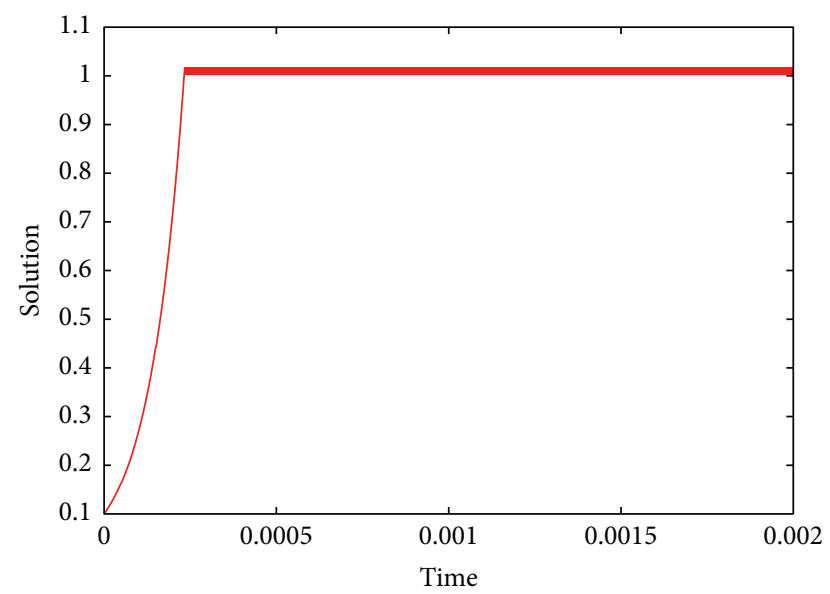

FIGURE 5: $\Delta t=2 \delta \varepsilon^{2} /(1-\delta)=0.0000020202020 \ldots$

3.6. Numerical Result of Corollary 10. In this subsection, we consider Corollary 10 numerically. To this end, we use the following initial data:

$$
u_{0}:=\frac{(1-\delta)^{5}}{(1+\delta)^{6}}=\frac{(1-0.01)^{5}}{(1+0.01)^{6}}=0.8958756 \ldots
$$

Then, we observe from Table 4 and Figure 8 that Corollary 10 holds. Namely, we observe that (47) holds with $n=6$.

3.7. Conclusion of ODE Problem $(D E)_{\delta}^{\varepsilon}$. From Theorem 7 and numerical experiments as above, we conclude that

(i) the mesh size of time $\Delta t$ must be smaller than $\delta \varepsilon^{2} /(1-$ $\delta$ ) in order to get the stable numerical experiments of $(D E)_{\delta}^{\varepsilon}$,

(ii) we have the stable numerical experiments of $(D E)_{\delta}^{\varepsilon}$ with the initial data $u_{0}^{\varepsilon}:=(1-\delta)^{n-1} /(1+\delta)^{n}$, even if the mesh size of time $\Delta t$ is equal to $2 \delta \varepsilon^{2} /(1-\delta)$.

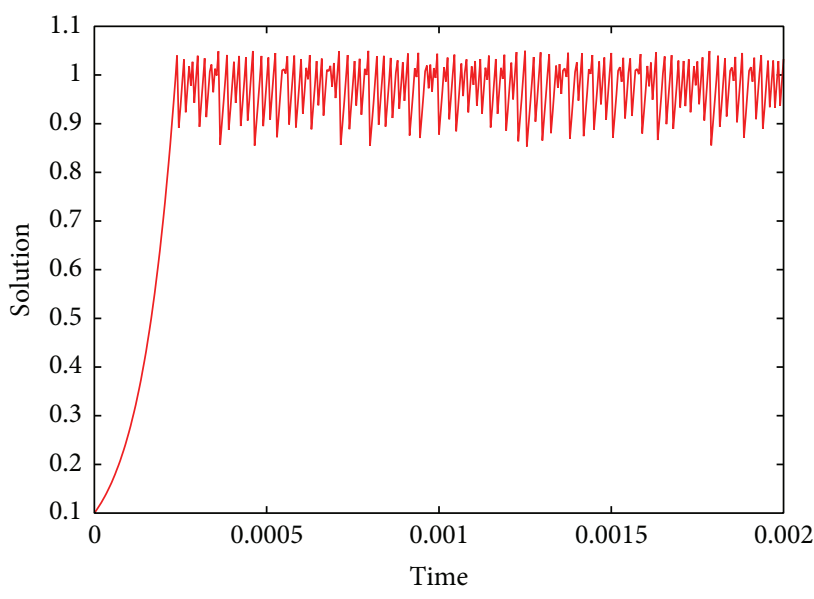

FIGURE 6: $2 \delta \varepsilon^{2} /(1-\delta)=0.0000020202020 \cdots<\Delta t=0.000005$.

TABLE 3: Numerical data: $\Delta t=2 \delta \varepsilon^{2} /(1-\delta)=0.0000020202020 \ldots$.

\begin{tabular}{lc}
\hline Number of iterations $i$ & The value of $u^{i}$ \\
\hline 0 & 0.100000 \\
1 & 0.102020 \\
2 & 0.104081 \\
3 & 0.106184 \\
4 & 0.108329 \\
5 & 0.110517 \\
$\vdots$ & $\vdots$ \\
110 & 0.902568 \\
111 & 0.920801 \\
112 & 0.939403 \\
113 & 0.958381 \\
114 & 0.977742 \\
115 & 0.997495 \\
116 & 1.017646 \\
117 & 1.002556 \\
118 & 1.017646 \\
119 & 1.002556 \\
120 & 1.017646 \\
121 & 1.002556 \\
122 & 1.017646 \\
123 & 1.002556 \\
124 & 1.017646 \\
125 & 1.002556 \\
126 & 1.017646 \\
127 & 1.002556 \\
128 & 1.017646 \\
129 & 1.002556 \\
130 & 1.017646 \\
\hline & \\
\hline &
\end{tabular}

\section{Solvability and Convergence Results for $(P)_{\delta}^{\varepsilon}$}

We begin by giving the rigorous definition of solutions to our PDE problem $(P)_{\delta}^{\varepsilon}(\varepsilon \in(0,1]$ and $\delta \in(0,1])$. 
TABLE 4: Numerical data: $\Delta t=2 \delta \varepsilon^{2} /(1-\delta)=0.0000020202020 \ldots$..

\begin{tabular}{lc}
\hline Number of iterations $i$ & The value of $u^{i}$ \\
\hline 0 & 0.895876 \\
1 & 0.913974 \\
2 & 0.932438 \\
3 & 0.951275 \\
4 & 0.970493 \\
5 & 0.990099 \\
6 & 1.010101 \\
7 & 1.010101 \\
8 & 1.010101 \\
9 & 1.010101 \\
10 & 1.010101 \\
\hline
\end{tabular}

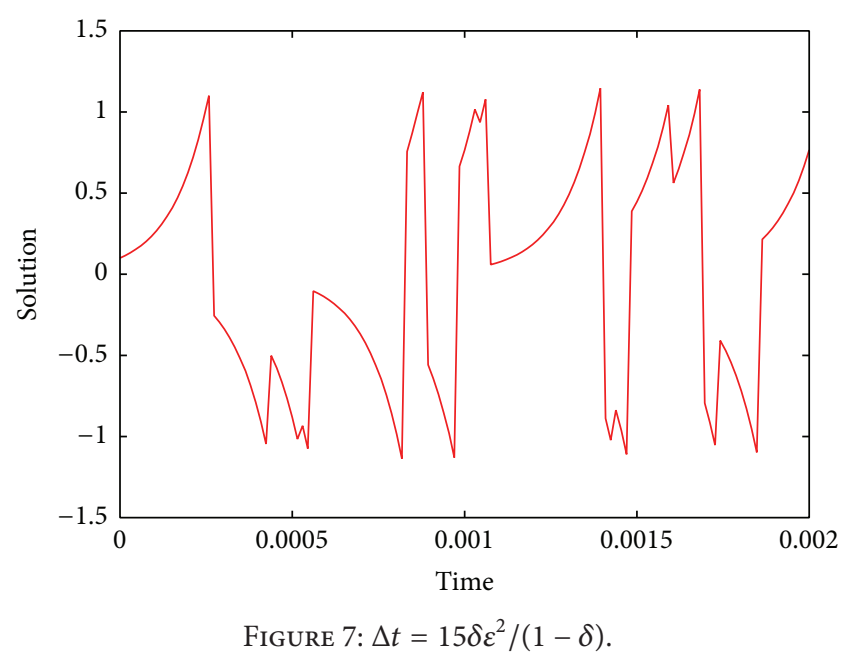

Definition 11. Let $\varepsilon \in(0,1], \delta \in(0,1]$, and $u_{0}^{\varepsilon} \in H$. Then, a function $u_{\delta}^{\varepsilon}:[0, T] \rightarrow H$ is called a solution to $(P)_{\delta}^{\varepsilon}$ on $[0, T]$, if the following conditions are satisfied:

(i) $u_{\delta}^{\varepsilon} \in W^{1,2}(0, T ; H) \cap L^{\infty}(0, T ; V)$.

(ii) The following variational identity holds:

$$
\begin{aligned}
\left(\left(u_{\delta}^{\varepsilon}\right)_{t}(t), z\right)_{H}+\left(\left(u_{\delta}^{\varepsilon}\right)_{x}(t), z_{x}\right)_{H} & \\
+\left(\frac{\left(\partial I_{[-1,1]}\right)_{\delta}\left(u_{\delta}^{\varepsilon}(t)\right)}{\varepsilon^{2}}, z\right)_{H} & =\left(\frac{u_{\delta}^{\varepsilon}(t)}{\varepsilon^{2}}, z\right)_{H} \\
\forall z & \in V \text {, a.e. } t \in(0, T) .
\end{aligned}
$$

(iii) $u_{\delta}^{\varepsilon}(0)=u_{0}^{\varepsilon}$ in $H$.

Now, we mention the solvability result of $(P)_{\delta}^{\varepsilon}$ on $[0, T]$.

Proposition 12. Let $\varepsilon \in(0,1]$ and $\delta \in(0,1]$. Assume the following condition:

$$
u_{0}^{\varepsilon} \in K:=\{z \in V ;-1 \leq z(x) \leq 1 \text { a.e. } x \in(0,1)\} .
$$

Then, for each $u_{0}^{\varepsilon} \in K$, there exists a unique solution $u_{\delta}^{\varepsilon}$ to $(P)_{\delta}^{\varepsilon}$ on $[0, T]$ in the sense of Definition 11.

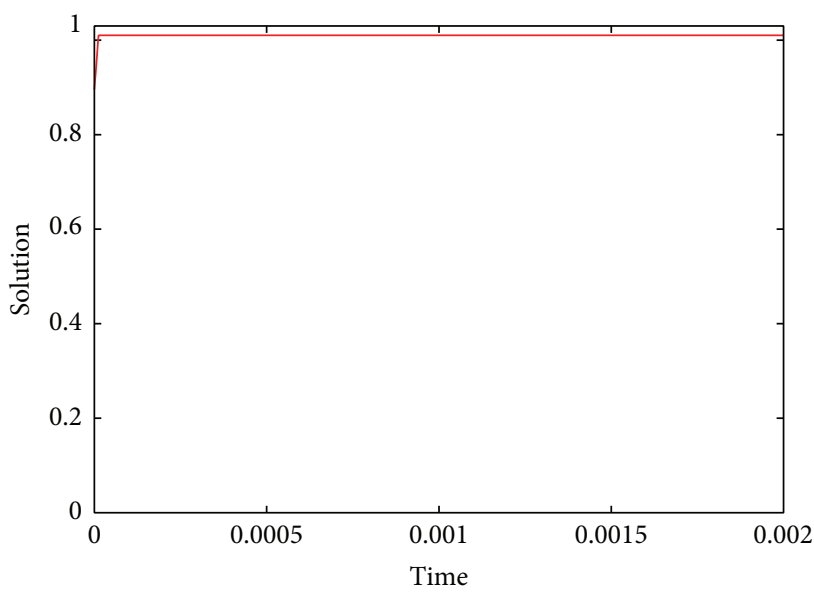

FIGURE 8: $\Delta t=2 \delta \varepsilon^{2} /(1-\delta)=0.0000020202020 \ldots$

Proof. By the same argument as in Proposition 2, we can show the existence-uniqueness of a solution $u_{\delta}^{\varepsilon}$ to $(P)_{\delta}^{\varepsilon}$ on $[0, T]$ for each $\varepsilon \in(0,1]$ and $\delta \in(0,1]$. Indeed, we can prove the uniqueness of solutions to $(P)_{\delta}^{\varepsilon}$ on $[0, T]$ by the quite standard arguments: monotonicity and Gronwall's inequality.

Also, we can show the existence of solutions to $(P)_{\delta}^{\varepsilon}$ on $[0, T]$ applying the abstract theory of evolution equations governed by subdifferentials. Indeed, we define a functional $\varphi_{\delta}^{\varepsilon}$ on $H$ by

$$
\begin{aligned}
& \varphi_{\delta}^{\varepsilon}(z) \\
& := \begin{cases}\frac{1}{2} \int_{0}^{1}\left|z_{x}\right|^{2} d x+\frac{1}{\varepsilon^{2}} \int_{0}^{1}\left(I_{[-1,1]}\right)_{\delta}(z(x)) d x, & \text { if } z \in V, \\
\infty, & \text { otherwise, }\end{cases}
\end{aligned}
$$

where $\left(I_{[-1,1]}\right)_{\delta}(\cdot)$ is the function defined in (14). Clearly, $\varphi_{\delta}^{\varepsilon}$ is proper, l.s.c., and convex on $H$ with the effective domain $D(\varphi)=V$.

Note that the problem $(P)_{\delta}^{\varepsilon}$ can be rewritten as in an abstract framework of the form

$$
(P P)_{\delta}^{\varepsilon} \begin{cases}\frac{d}{d t} u_{\delta}^{\varepsilon}(t)+\partial \varphi_{\delta}^{\varepsilon}\left(u_{\delta}^{\varepsilon}(t)\right)-\frac{1}{\varepsilon^{2}} u_{\delta}^{\varepsilon}(t)=0 & \text { in } H, \text { for } t>0 \\ u^{\varepsilon}(0)=u_{0}^{\varepsilon} & \text { in } H .\end{cases}
$$

Therefore, applying the Lipschitz perturbation theory of abstract evolution equations (cf. [23-25]), we can show the existence of a solution $u_{\delta}^{\varepsilon}$ to $(P P)_{\delta}^{\varepsilon}$, hence, $(P)_{\delta}^{\varepsilon}$, on $[0, T]$ for each $\varepsilon \in(0,1]$ and $\delta \in(0,1]$ in the sense of Definition 11 . Thus, the proof of Proposition 12 has been completed.

Next, we recall the convergence result of $(P)_{\delta}^{\varepsilon}$ as $\delta \rightarrow 0$. Taking into account Lemma 4 (cf. (18)), we observe that the following lemma holds. 
Lemma 13 (cf. [27, Section 5], [22, Chapter 2], [28, Section $2])$. Let $\varepsilon \in(0,1]$, and define a functional $\varphi^{\varepsilon}$ on $H$ by

$$
\begin{aligned}
& \varphi^{\varepsilon}(z) \\
& := \begin{cases}\frac{1}{2} \int_{0}^{1}\left|z_{x}\right|^{2} d x+\frac{1}{\varepsilon^{2}} \int_{0}^{1} I_{[-1,1]}(z(x)) d x, & \text { if } z \in V, \\
\infty, & \text { otherwise. }\end{cases}
\end{aligned}
$$

Then,

$$
\begin{aligned}
\varphi_{\delta}^{\varepsilon}(\cdot) & \longrightarrow \varphi^{\varepsilon}(\cdot) \\
& \text { on } H \text { in the sense of Mosco [26] as } \delta \longrightarrow 0 .
\end{aligned}
$$

By Lemma 13 and the general convergence theory of evolution equations, we get the following result.

Proposition 14 (cf. [27, Section 5], [28, Section 2]). Let $\varepsilon \in$ $(0,1], \delta \in(0,1]$, and $u_{0}^{\varepsilon} \in K$. Also, let $u_{\delta}^{\varepsilon}$ be the unique solution to $(P)_{\delta}^{\varepsilon}$ on $[0, T]$. Then, $u_{\delta}^{\varepsilon}$ converges to the unique function $u^{\varepsilon}$ to $(P)^{\varepsilon}$ on $[0, T]$ in the sense that

$$
u_{\delta}^{\varepsilon} \longrightarrow u^{\varepsilon} \quad \text { strongly in } C([0, T] ; H) \text { as } \delta \longrightarrow 0 \text {. }
$$

Proof. Note that the problem $(P)^{\varepsilon}$ can be rewritten as in an abstract framework of the form

$$
(P P)^{\varepsilon} \begin{cases}\frac{d}{d t} u^{\varepsilon}(t)+\partial \varphi^{\varepsilon}\left(u^{\varepsilon}(t)\right)-\frac{1}{\mathcal{E}^{2}} u^{\varepsilon}(t) \ni 0 & \text { in } H, \text { for } t>0, \\ u^{\varepsilon}(0)=u_{0}^{\varepsilon} & \text { in } H .\end{cases}
$$

Therefore, by Lemma 13 and the abstract convergence theory of evolution equations (cf. [27, 28]), we observe that the solution $u_{\delta}^{\varepsilon}$ to $(P P)_{\delta}^{\varepsilon}$ converges to the unique solution $u^{\varepsilon}$ to $(P P)^{\varepsilon}$ on $[0, T]$ as $\delta \rightarrow 0$ in the sense of $(60)$. Note that $u^{\varepsilon}$ (resp., $u_{\delta}^{\varepsilon}$ ) is the unique solution to $(P)^{\varepsilon}$ (resp., $\left.(P)_{\delta}^{\varepsilon}\right)$ on $[0, T]$ (cf. Proposition 12). Thus, we conclude that Proposition 14 holds.

\section{Stable Criteria and Numerical Experiments for $(P)_{\delta}^{\varepsilon}$}

In this section we consider $(P)_{\delta}^{\varepsilon}$ from the viewpoint of numerical analysis.

Remark 15. Note from Proposition 14 that $(P)_{\delta}^{\varepsilon}$ is the approximating problem of $(P)^{\varepsilon}$. Also note from (5) that the constraint $\partial I_{[-1,1]}(\cdot)$ is the multivalued function. Therefore, it is very difficult to study $(P)^{\varepsilon}$ numerically.

In order to perform the numerical experiments of $(P)_{\delta}^{\varepsilon}$, we consider the following explicit finite difference scheme to $(P)_{\delta}^{\varepsilon}$, denoted by $(D P)_{\delta}^{\varepsilon}$ :

$$
(D P)_{\delta}^{\varepsilon}\left\{\begin{array}{lc}
\frac{u_{k}^{n+1}-u_{k}^{n}}{\Delta t}-\frac{u_{k-1}^{n}-2 u_{k}^{n}+u_{k+1}^{n}}{(\Delta x)^{2}}+\frac{\left(\partial I_{[-1,1]}\right)_{\delta}\left(u_{k}^{n}\right)}{\varepsilon^{2}}=\frac{u_{k}^{n}}{\varepsilon^{2}} & \text { for } n=0,1,2, \ldots, N_{t}, k=1,2, \ldots, N_{x}-1 \\
u_{0}^{n}=u_{1}^{n}, u_{N_{x}}^{n}=u_{N_{x}-1}^{n} & \text { for } n=1,2, \ldots, N_{t} \\
u_{k}^{0}=u_{0}^{\varepsilon}\left(x_{k}\right) & \text { for } k=0,1,2, \ldots, N_{x}
\end{array}\right.
$$

where $\Delta t$ is the mesh size of time, $\Delta x$ is the mesh size of space, $N_{t}$ is the integer part of number $T / \Delta t, N_{x}$ is the integer part of number $1 / \Delta x$, and $x_{k}:=k \Delta x$.

We observe that $u_{k}^{n}$ is the approximating solution of $(P)_{\delta}^{\varepsilon}$ at the time $t_{n}:=n \Delta t$ and the position $x_{k}:=k \Delta x$. Also, we observe that the explicit finite difference scheme $(D P)_{\delta}^{\varepsilon}$ converges to $(P)_{\delta}^{\varepsilon}$ as $\Delta t \rightarrow 0$ and $\Delta x \rightarrow 0$.

From Figure 1, we observe that we have to choose the suitable constants $\varepsilon$ and $\delta$ and the mesh size of time $\Delta t$ and the mesh size of space $\Delta x$ in order to get the stable numerical results of $(D P)_{\delta}^{\varepsilon}$. Now, let us mention our second main result concerning the stability of $(D E)_{\delta}^{\varepsilon}$.

Theorem 16. Let $\varepsilon \in(0,1], \delta \in(0,1), \Delta t \in(0,1], \Delta x \in(0,1]$, $T>0$, and $u_{0}^{\varepsilon} \in K$, where $K$ is the set of initial values defined in Proposition 12 ( $c f$. (A)). Let $N_{x}$ be the integer part of number $1 / \Delta x$, and let $\left\{u_{k}^{n} ; n \geq 0, k=0,1, \ldots, N_{x}\right\}$ be the solution to $(D P)_{\delta}^{\varepsilon}$. Also, let $c_{0} \in(0,1)$ and assume that

$$
0<\Delta t \leq \frac{c_{0} \delta \varepsilon^{2}}{1-\delta}
$$

$$
0 \leq \frac{\Delta t}{(\Delta x)^{2}} \leq \frac{1-c_{0}}{2}
$$

Then, one has the following:

(i) The solution to $(D P)_{\delta}^{\varepsilon}$ is bounded in the following sense:

$$
\max _{0 \leq k \leq N_{x}}\left|u_{k}^{n}\right| \leq \frac{1}{1-\delta} \quad \forall n \geq 0 .
$$

(ii) $\left\{u_{k}^{n} ; k=0,1, \ldots, N_{x}\right\}$ does not oscillate with respect to $n \geq 0$.

Proof. We first show (i), that is, (64), by the mathematical induction.

Clearly (64) holds for $n=0$ because of $u_{0}^{\varepsilon} \in K$.

Now, we assume that

$$
\max _{0 \leq k \leq N_{x}}\left|u_{k}^{i}\right| \leq \frac{1}{1-\delta} \quad \forall i=0,1, \ldots, n .
$$


We observe that the explicit finite difference problem $(D P)_{\delta}^{\varepsilon}$ can be reformulated as in the following form:

$$
\begin{array}{r}
u_{k}^{n+1}=r u_{k-1}^{n}+r u_{k+1}^{n}+(1-2 r) u_{k}^{n}-\frac{\Delta t}{\varepsilon^{2}} f_{\delta}\left(u_{k}^{n}\right) \\
\forall n=0,1,2, \ldots, N_{t}, \quad k=1,2, \ldots, N_{x}-1,
\end{array}
$$

where we put $r:=\Delta t /(\Delta x)^{2}$ and $f_{\delta}(\cdot)$ is the function defined by (23).

We observe from (63), (65), and (66) that

$$
\begin{aligned}
& \frac{1}{1-\delta}-u_{k}^{n+1}= r\left(\frac{1}{1-\delta}-u_{k-1}^{n}\right)+r\left(\frac{1}{1-\delta}-u_{k+1}^{n}\right) \\
&+(1-2 r)\left(\frac{1}{1-\delta}-u_{k}^{n}\right)+\frac{\Delta t}{\varepsilon^{2}} f_{\delta}\left(u_{k}^{n}\right) \\
& \geq(1-2 r)\left(\frac{1}{1-\delta}-u_{k}^{n}\right)+\frac{\Delta t}{\varepsilon^{2}} f_{\delta}\left(u_{k}^{n}\right) \\
& \forall k=1,2, \ldots, N_{x}-1 .
\end{aligned}
$$

From (23), (63), and (65) we infer that the function $[-1 /(1-\delta), 1 /(1-\delta)] \ni z \rightarrow(1-2 r)(1 /(1-\delta)-z)+\Delta t / \varepsilon^{2} f_{\delta}(z)$ is nonnegative and continuous. Indeed, it follows from (23) that the function $[-1 /(1-\delta), 1] \ni z \rightarrow(1-2 r)(1 /(1-\delta)-$ $z)+\Delta t / \varepsilon^{2} f_{\delta}(z)$ attains a minimum value at $z=1$. Therefore, we observe from (23) and (63) that

$$
\begin{aligned}
& (1-2 r)\left(\frac{1}{1-\delta}-z\right)+\frac{\Delta t}{\varepsilon^{2}} f_{\delta}(z) \\
& \geq(1-2 r)\left(\frac{1}{1-\delta}-1\right)+\frac{\Delta t}{\varepsilon^{2}} f_{\delta}(1) \\
& =(1-2 r) \cdot \frac{\delta}{1-\delta}-\frac{\Delta t}{\varepsilon^{2}} \geq \frac{c_{0} \delta}{1-\delta}-\frac{\Delta t}{\varepsilon^{2}} \geq 0 \\
& \forall z \in\left[-\frac{1}{1-\delta}, 1\right]
\end{aligned}
$$

Also, for any $z \in[1,1 /(1-\delta)]$, we observe from (23) that

$$
\begin{aligned}
(1 & -2 r)\left(\frac{1}{1-\delta}-z\right)+\frac{\Delta t}{\varepsilon^{2}} f_{\delta}(z) \\
& =(1-2 r)\left(\frac{1}{1-\delta}-z\right)+\frac{\Delta t}{\varepsilon^{2}} \cdot\left(\frac{z-1}{\delta}-z\right) \\
& =\left[\frac{1-\delta}{\delta \varepsilon^{2}} \Delta t-(1-2 r)\right] z+(1-2 r) \frac{1}{1-\delta}-\frac{\Delta t}{\delta \varepsilon^{2}}
\end{aligned}
$$

Here we note from (63) that

$$
\frac{1-\delta}{\delta \varepsilon^{2}} \Delta t-(1-2 r) \leq \frac{1-\delta}{\delta \varepsilon^{2}} \Delta t-c_{0} \leq 0 .
$$

Therefore, we infer from $(69)$ that the function $[1,1 /(1-\delta)] \ni$ $z \rightarrow(1-2 r)(1 /(1-\delta)-z)+\Delta t / \varepsilon^{2} f_{\delta}(z)$ is nonincreasing and attains a minimum value at $z=1 /(1-\delta)$. Hence, we have

$$
\begin{array}{r}
(1-2 r)\left(\frac{1}{1-\delta}-z\right)+\frac{\Delta t}{\varepsilon^{2}} f_{\delta}(z) \geq \frac{\Delta t}{\varepsilon^{2}} f_{\delta}\left(\frac{1}{1-\delta}\right)=0 \\
\forall z \in\left[1, \frac{1}{1-\delta}\right] .
\end{array}
$$

Thus, we observe from (68) and (71) that

$$
\begin{aligned}
(1-2 r)\left(\frac{1}{1-\delta}-z\right)+\frac{\Delta t}{\varepsilon^{2}} f_{\delta}(z) & \geq 0, \\
\forall z & \in\left[-\frac{1}{1-\delta}, \frac{1}{1-\delta}\right],
\end{aligned}
$$

which implies from (65) and (67) that

$$
\frac{1}{1-\delta}-u_{k}^{n+1} \geq 0 \quad \forall k=1,2, \ldots, N_{x}-1
$$

Similarly, we observe from (63), (65), and (66) that

$$
\begin{array}{r}
u_{k}^{n+1}+\frac{1}{1-\delta}= \\
+(1-2 r)\left(u_{k-1}^{n}+\frac{1}{1-\delta}\right)+r\left(u_{k+1}^{n}+\frac{1}{1-\delta}\right)-\frac{\Delta t}{\varepsilon^{2}} f_{\delta}\left(u_{k}^{n}\right) \\
\geq(1-2 r)\left(u_{k}^{n}+\frac{1}{1-\delta}\right)-\frac{\Delta t}{\varepsilon^{2}} f_{\delta}\left(u_{k}^{n}\right) \\
\forall k=1,2, \ldots, N_{x}-1 .
\end{array}
$$

By similar arguments as above, we observe that the function $[-1 /(1-\delta), 1 /(1-\delta)] \ni z \rightarrow(1-2 r)(z+1 /(1-\delta))-\Delta t / \varepsilon^{2} f_{\delta}(z)$ is nonnegative and continuous. In fact, it follows from (23) that the function $[-1,1 /(1-\delta)] \ni z \rightarrow(1-2 r)(z+1 /(1-\delta))-$ $\Delta t / \varepsilon^{2} f_{\delta}(z)$ attains a minimum value at $z=-1$. Therefore, we observe from (23) and (63) that

$$
\begin{aligned}
& (1-2 r)\left(z+\frac{1}{1-\delta}\right)-\frac{\Delta t}{\varepsilon^{2}} f_{\delta}(z) \\
& \geq(1-2 r)\left(-1+\frac{1}{1-\delta}\right)-\frac{\Delta t}{\varepsilon^{2}} f_{\delta}(-1) \\
& =(1-2 r) \cdot \frac{\delta}{1-\delta}-\frac{\Delta t}{\varepsilon^{2}} \geq \frac{c_{0} \delta}{1-\delta}-\frac{\Delta t}{\varepsilon^{2}} \geq 0 \\
& \forall z \in\left[-1, \frac{1}{1-\delta}\right] .
\end{aligned}
$$

Also, for any $z \in[-1 /(1-\delta),-1]$, we observe from (23) that

$$
\begin{aligned}
(1 & -2 r)\left(z+\frac{1}{1-\delta}\right)-\frac{\Delta t}{\varepsilon^{2}} f_{\delta}(z) \\
& =(1-2 r)\left(z+\frac{1}{1-\delta}\right)-\frac{\Delta t}{\varepsilon^{2}} \cdot\left(\frac{1+z}{\delta}-z\right) \\
& =\left[(1-2 r)-\frac{1-\delta}{\delta \varepsilon^{2}} \Delta t\right] z+(1-2 r) \frac{1}{1-\delta}-\frac{\Delta t}{\delta \varepsilon^{2}} .
\end{aligned}
$$

Here we note from (63) that

$$
(1-2 r)-\frac{1-\delta}{\delta \varepsilon^{2}} \Delta t \geq c_{0}-\frac{1-\delta}{\delta \varepsilon^{2}} \Delta t \geq 0 .
$$

Therefore, we infer from (76) that the function $[-1 /(1-$ $\delta),-1] \quad \ni z \rightarrow(1-2 r)(z+1 /(1-\delta))-\Delta t / \varepsilon^{2} f_{\delta}(z)$ is nondecreasing and attains a minimum value at $z=-1 /(1-\delta)$. Hence, we have

$$
\begin{aligned}
& (1-2 r)\left(z+\frac{1}{1-\delta}\right)-\frac{\Delta t}{\varepsilon^{2}} f_{\delta}(z) \geq-\frac{\Delta t}{\varepsilon^{2}} f_{\delta}\left(-\frac{1}{1-\delta}\right) \\
& =0 \quad \forall z \in\left[-\frac{1}{1-\delta},-1\right] .
\end{aligned}
$$


Thus, we observe from (75) and (78) that

$$
\begin{aligned}
(1-2 r)\left(z+\frac{1}{1-\delta}\right)-\frac{\Delta t}{\varepsilon^{2}} f_{\delta}(z) & \geq 0 \\
\forall z & \in\left[-\frac{1}{1-\delta}, \frac{1}{1-\delta}\right],
\end{aligned}
$$

which implies from (65) and (74) that

$$
u_{k}^{n+1}+\frac{1}{1-\delta} \geq 0 \quad \forall k=1,2, \ldots, N_{x}-1 .
$$

Taking into account Neumann boundary condition, namely, by $u_{0}^{n+1}=u_{1}^{n+1}$ and $u_{N_{x}}^{n+1}=u_{N_{x}-1}^{n+1}$, we observe from (73) and (80) that

$$
\max _{0 \leq k \leq N_{x}}\left|u_{k}^{n+1}\right| \leq \frac{1}{1-\delta}
$$

which implies that (65) holds for $i=n+1$. Therefore, we conclude from the mathematical induction that (64) holds. Hence, the proof of (i) of Theorem 16 has been completed.

Next, we show (ii) by the standard arguments. Namely, we reformulate $(D P)_{\delta}^{\varepsilon}$ as in the following form:

$$
\begin{aligned}
& \left(\begin{array}{c}
u_{1}^{n+1} \\
u_{2}^{n+1} \\
\vdots \\
u_{N_{x}-1}^{n+1}
\end{array}\right) \\
& =\left(\begin{array}{cccccc}
1-2 r & r & & & & \\
r & 1-2 r & r & & & \\
& r & 1-2 r & r & & \\
& & \ddots & \ddots & & \\
& & & r & 1-2 r & r \\
& & & & r & 1-2 r
\end{array}\right)\left(\begin{array}{c}
u_{1}^{n} \\
u_{2}^{n} \\
\vdots \\
u_{N_{x}-1}^{n}
\end{array}\right) \\
& +r\left(\begin{array}{c}
u_{0}^{n} \\
0 \\
\vdots \\
0 \\
u_{N_{x}}^{n}
\end{array}\right)+\left(\begin{array}{c}
-\frac{\Delta t}{\varepsilon^{2}} f_{\delta}\left(u_{1}^{n}\right) \\
-\frac{\Delta t}{\varepsilon^{2}} f_{\delta}\left(u_{2}^{n}\right) \\
\vdots \\
-\frac{\Delta t}{\varepsilon^{2}} f_{\delta}\left(u_{N_{x}-1}^{n}\right)
\end{array}\right) .
\end{aligned}
$$

Here by taking into account Neumann boundary condition and initial condition, namely, by $u_{0}^{n}=u_{1}^{n}$ and $u_{N_{x}}^{n}=u_{N_{x}-1}^{n}$ for all $n \geq 0$, we observe that (82) is reformulated as in the following form:

$$
\vec{u}^{(n+1)}=A \vec{u}^{(n)}+\left(\begin{array}{c}
r u_{1}^{n}-\frac{\Delta t}{\varepsilon^{2}} f_{\delta}\left(u_{1}^{n}\right) \\
-\frac{\Delta t}{\varepsilon^{2}} f_{\delta}\left(u_{2}^{n}\right) \\
\vdots \\
r u_{N_{x}-1}^{n}-\frac{\Delta t}{\varepsilon^{2}} f_{\delta}\left(u_{N_{x}-1}^{n}\right)
\end{array}\right),
$$

where we put

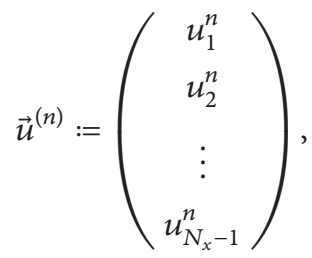

A

$$
=\left(\begin{array}{cccccc}
1-2 r & r & & & & \\
r & 1-2 r & r & & & \\
& r & 1-2 r & r & & \\
& & \ddots & \ddots & & \\
& & & r & 1-2 r & r \\
& & & & r & 1-2 r
\end{array}\right) .
$$

Note from (23) that

$$
f_{\delta}\left(u_{k}^{n}\right)= \begin{cases}\frac{1-\delta}{\delta} u_{k}^{n}+\frac{1}{\delta} & \text { if } u_{k}^{n} \leq-1, \\ -u_{k}^{n} & \text { if } u_{k}^{n} \in[-1,1] \\ \frac{1-\delta}{\delta} u_{k}^{n}-\frac{1}{\delta} & \text { if } u_{k}^{n} \geq 1 .\end{cases}
$$

Defining

$$
\begin{aligned}
& b_{k}^{n}:= \begin{cases}-1 & \text { if } u_{k}^{n} \in[-1,1], \\
\frac{1-\delta}{\delta} & \text { if } u_{k}^{n} \notin[-1,1],\end{cases} \\
& \widetilde{b}_{k}^{n}:= \begin{cases}\frac{1}{\delta} & \text { if } u_{k}^{n} \leq-1, \\
0 & \text { if } u_{k}^{n} \in[-1,1], \\
-\frac{1}{\delta} & \text { if } u_{k}^{n} \geq 1,\end{cases}
\end{aligned}
$$

we observe from (85) and (86) that

$$
\left(\begin{array}{c}
r u_{1}^{n}-\frac{\Delta t}{\varepsilon^{2}} f_{\delta}\left(u_{1}^{n}\right) \\
-\frac{\Delta t}{\varepsilon^{2}} f_{\delta}\left(u_{2}^{n}\right) \\
\vdots \\
r u_{N_{x}-1}^{n}-\frac{\Delta t}{\varepsilon^{2}} f_{\delta}\left(u_{N_{x}-1}^{n}\right)
\end{array}\right)
$$




$$
\begin{aligned}
& =\left(\begin{array}{cccc}
r-\frac{\Delta t}{\varepsilon^{2}} b_{1}^{n} & & & \\
& -\frac{\Delta t}{\varepsilon^{2}} b_{2}^{n} & & \\
& & \ddots & \\
& & & r-\frac{\Delta t}{\varepsilon^{2}} b_{N_{x}-1}^{n}
\end{array}\right)\left(\begin{array}{c}
u_{1}^{n} \\
u_{2}^{n} \\
\vdots \\
u_{N_{x}-1}^{n}
\end{array}\right) \\
& +\left(\begin{array}{c}
-\frac{\Delta t}{\varepsilon^{2}} \widetilde{b}_{1}^{n} \\
-\frac{\Delta t}{\varepsilon^{2}} \widetilde{b}_{2}^{n} \\
\vdots \\
-\frac{\Delta t}{\mathcal{E}^{2}} \widetilde{b}_{N_{x}-1}^{n}
\end{array}\right) .
\end{aligned}
$$

Using the matrix as above, (82) can be rewritten as in the following form:

$$
\vec{u}^{(n+1)}=A \vec{u}^{(n)}+B \vec{u}^{(n)}+\widetilde{\vec{b}}^{(n)}, \quad(n \geq 0),
$$

where we define

$$
\begin{aligned}
B & :=\left(\begin{array}{ccc}
r-\frac{\Delta t}{\varepsilon^{2}} b_{1}^{n} & & \\
& -\frac{\Delta t}{\varepsilon^{2}} b_{2}^{n} & \\
& \ddots & \\
\widetilde{\vec{b}}^{(n)}:=\left(\begin{array}{cc}
-\frac{\Delta t}{\varepsilon^{2}} \widetilde{b}_{1}^{n} \\
-\frac{\Delta t}{\varepsilon^{2}} \widetilde{b}_{2}^{n} \\
\vdots \\
-\frac{\Delta t}{\varepsilon^{2}} \widetilde{b}_{N_{x}-1}^{n}
\end{array}\right), & \\
& r-
\end{array}\right) .
\end{aligned}
$$

By the general theory, we observe that the eigenvalue $\lambda_{j}$ of matrix $A$ is given by

$$
\lambda_{j}:=1-4 r \sin ^{2}\left(\frac{j \pi}{2 N_{x}}\right) \text { for } j=1,2, \ldots, N_{x}-1 \text {, }
$$

which implies that $\lambda_{1}$ (resp., $\lambda_{N_{x}-1}$ ) is the maximum (resp., minimum) eigenvalue of $A$.

Now let $\left\{\widetilde{\lambda}_{j} ; j=1,2, \ldots, N_{x}-1\right\}$ be the set of all eigenvalues of matrix $\widetilde{A}:=A+B$ such that

$$
\tilde{\lambda}_{1} \geq \tilde{\lambda}_{2} \geq \cdots \geq \tilde{\lambda}_{N_{x}-1}
$$

Also, let $\left\{\lambda_{j}^{B} ; j=1,2, \ldots, N_{x}-1\right\}$ be the set of all eigenvalues of $B$ such that

$$
\lambda_{1}^{B} \geq \lambda_{2}^{B} \geq \cdots \geq \lambda_{N_{x}-1}^{B}
$$

Then, by the abstract perturbation theory of matrix, we observe that

$$
\lambda_{N_{x}-1}+\lambda_{j}^{B} \leq \tilde{\lambda}_{j} \leq \lambda_{1}+\lambda_{j}^{B}, \quad \forall j=1,2, \ldots, N_{x}-1
$$

Since $B$ is the symmetric matrix, it follows from (63), (86), and (90) that

$$
\begin{aligned}
& \lambda_{N_{x}-1}+\lambda_{j}^{B} \geq 1-4 r \sin ^{2}\left(\frac{\left(N_{x}-1\right) \pi}{2 N_{x}}\right)-\frac{\Delta t}{\varepsilon^{2}} \cdot \frac{1-\delta}{\delta} \\
& \geq 1-4 r-\frac{\Delta t(1-\delta)}{\delta \varepsilon^{2}} \\
& \geq 1-4 \cdot \frac{1-c_{0}}{2}-c_{0}=-1+c_{0}>-1 \\
& \forall j=1,2, \ldots, N_{x}-1 .
\end{aligned}
$$

Thus, we conclude from (93) and the above inequality that

$$
\tilde{\lambda}_{j}>-1 \quad \forall j=1,2, \ldots, N_{x}-1
$$

Note that (95) holds in any case: $u_{k}^{n} \in[-1,1]$ and $u_{k}^{n} \notin[-1,1]$.

Next, we now assume $\max _{1 \leq k \leq N_{x}-1}\left|u_{k}^{n}\right| \leq 1$. Then, we observe from (63) and (86) that

$$
\begin{aligned}
& 1-2 r-\frac{\Delta t}{\varepsilon^{2}} b_{k}^{n}=1-2 \frac{\Delta t}{(\Delta x)^{2}}+\frac{\Delta t}{\varepsilon^{2}} \\
& \geq 1-2 \cdot \frac{1-c_{0}}{2}+\frac{\Delta t}{\varepsilon^{2}}>0 \\
& \forall k=1,2, \ldots, N_{x}-1 .
\end{aligned}
$$

Therefore, all components of $A+B$ are positive. Hence, the sum of all components in the $k$ th row of $A+B$ is the following:

$$
\begin{aligned}
& \left|1-r-\frac{\Delta t}{\varepsilon^{2}} b_{k}^{n}\right|+|r|=1-r-\frac{\Delta t}{\varepsilon^{2}} b_{k}^{n}+r=1+\frac{\Delta t}{\varepsilon^{2}}>1 \\
& \quad \text { for } k=1 \text { or } N_{x}-1, \\
& |r|+\left|1-2 r-\frac{\Delta t}{\varepsilon^{2}} b_{k}^{n}\right|+|r|=r+1-2 r-\frac{\Delta t}{\varepsilon^{2}} b_{k}^{n}+r \\
& =1+\frac{\Delta t}{\varepsilon^{2}}>1 \quad \forall k=2,3, \ldots, N_{x}-2 .
\end{aligned}
$$

Therefore, we observe from (97) that $\max _{1 \leq k \leq N_{x}-1}\left|u_{k}^{n}\right|$ is increasing with respect to $n$ in the case when $\max _{1 \leq k \leq N_{x}-1}\left|u_{k}^{n}\right| \leq 1$.

However, if $u_{k}^{n} \notin[-1,1]$ for some $k=1,2, \ldots, N_{x}-1$, it follows from (63) and (86) that

$$
\begin{aligned}
1-2 r-\frac{\Delta t}{\varepsilon^{2}} b_{k}^{n} & =1-2 \frac{\Delta t}{(\Delta x)^{2}}-\frac{\Delta t}{\varepsilon^{2}} \cdot \frac{1-\delta}{\delta} \\
& \geq 1-2 \cdot \frac{1-c_{0}}{2}-c_{0}=0 .
\end{aligned}
$$


Therefore, the sum of all components in the $k$ th row of $A+B$ is the following:

$$
\begin{aligned}
& \left|1-r-\frac{\Delta t}{\varepsilon^{2}} b_{k}^{n}\right|+|r|=1-r-\frac{\Delta t}{\varepsilon^{2}} b_{k}^{n}+r \\
& =1-\frac{\Delta t}{\varepsilon^{2}} \cdot \frac{1-\delta}{\delta}<1 \\
& \quad \text { if } u_{k}^{n} \notin[-1,1] \text { for some } k=1 \text { or } N_{x}-1, \\
& |r|+\left|1-2 r-\frac{\Delta t}{\varepsilon^{2}} b_{k}^{n}\right|+|r|=r+1-2 r-\frac{\Delta t}{\varepsilon^{2}} b_{k}^{n}+r \\
& =1-\frac{\Delta t}{\varepsilon^{2}} \cdot \frac{1-\delta}{\delta}<1 \\
& \quad \text { if } u_{k}^{n} \notin[-1,1] \text { for some } k=2,3, \ldots, N_{x}-2 .
\end{aligned}
$$

Although $\max _{1 \leq k \leq N_{x}-1}\left|u_{k}^{n}\right|$ is increasing with respect to $n$ in the case when $\max _{1 \leq k \leq N_{x}-1}\left|u_{k}^{n}\right| \leq 1$ (cf. (97)), we conclude from (95) and (99) that (ii) of Theorem 16 holds. Thus, the proof of Theorem 16 has been completed.

Remark 17. By (63) we get the suitable mesh size of space $\Delta x$. In fact, for each $\varepsilon \in(0,1], \delta \in(0,1)$ we take the constant $\widetilde{c}_{0} \in(0,1), \Delta t \in(0,1], \Delta x \in(0,1]$ such that

$$
\begin{aligned}
\Delta t & \leq \frac{\widetilde{c}_{0} \delta \varepsilon^{2}}{1-\delta}, \\
\frac{\Delta t}{(\Delta x)^{2}} & =\frac{1-\widetilde{c}_{0}}{2} .
\end{aligned}
$$

Then, we have

$$
\Delta t=\frac{1-\widetilde{c}_{0}}{2} \cdot(\Delta x)^{2} \leq \frac{\widetilde{c}_{0} \delta \varepsilon^{2}}{1-\delta} .
$$

Thus, we have the following condition of $\Delta x$ :

$$
0<\Delta x<\varepsilon \sqrt{\frac{2 \widetilde{c}_{0} \delta}{\left(1-\widetilde{c}_{0}\right)(1-\delta)}} .
$$

Remark 18. We can take $c_{0}=0$ in (63) for the explicit finite difference scheme to the following usual heat equation with Neumann boundary condition:

$$
\begin{aligned}
& u_{t}^{\varepsilon}-u_{x x}^{\varepsilon}=0 \quad \text { in } Q:=(0, T) \times(0,1), \\
& u_{x}^{\varepsilon}(t, 0)=u_{x}^{\varepsilon}(t, 1)=0, \quad t \in(0, T), \\
& u^{\varepsilon}(0, x)=u_{0}^{\varepsilon}(x), \quad x \in(0,1) .
\end{aligned}
$$

Taking into account Theorem 16, we provide the numerical experiments of $(D P)_{\delta}^{\varepsilon}$ as follows. To this end, we use the following numerical data.

\section{Numerical Data of $(D P)_{\delta}^{\varepsilon}$}

The numerical data is $T=0.01, \delta=0.01, \Delta x=0.005$, $c_{0}=0.6$.

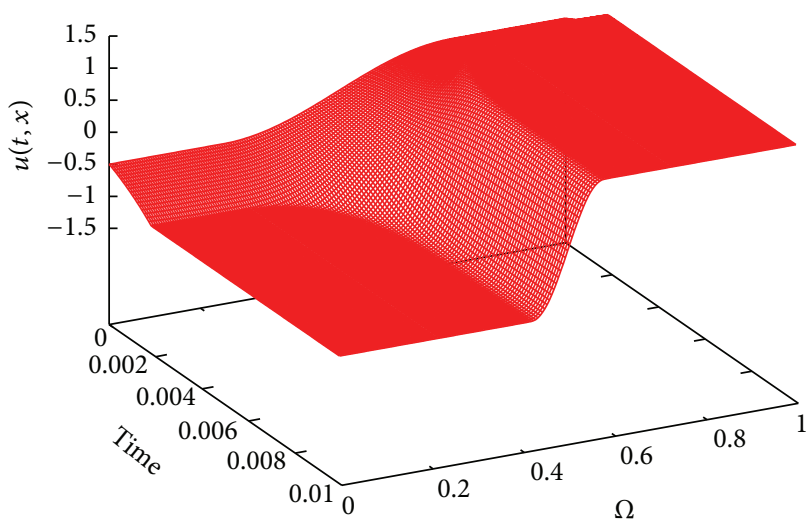

FIGURE 9: $\varepsilon=0.05, \Delta t=0.000005, \Delta x=0.005$, and $\delta=0.01$.

Also, we consider the initial data $u_{0}^{\varepsilon}(x)$ defined by

$$
u_{0}^{\varepsilon}(x)= \begin{cases}-0.5, & \text { if } x \in[0.00,0.25], \\ -0.5 \sin (2 \pi x), & \text { if } x \in[0.25,0.75], \\ 0.5, & \text { if } x \in[0.75,1.00]\end{cases}
$$

5.1. The Case When $\varepsilon=0.05$ and $\Delta t=0.000005$. Now, we consider the case when $\varepsilon=0.05$ and $\Delta t=0.000005$. In this case, we observe that

$$
\begin{aligned}
\frac{\Delta t}{(\Delta x)^{2}} & =\frac{0.000005}{(0.005)^{2}}=0.2=\frac{1-c_{0}}{2}, \\
\frac{c_{0} \delta \varepsilon^{2}}{1-\delta} & =\frac{0.6 \times 0.01 \times(0.05)^{2}}{1-0.01} \\
& =0.00001515151515 \ldots
\end{aligned}
$$

Therefore, we have

$$
\frac{c_{0} \delta \varepsilon^{2}}{1-\delta}=0.00001515151515 \cdots>\Delta t,
$$

which implies that criteria condition (63) holds. Thus, we can provide the stable numerical experiment of $(D P)_{\delta}^{\varepsilon}$. Indeed, we provide Figure 9, which implies the stable numerical experiment of $(D P)_{\delta}^{\varepsilon}$.

5.2. The Case When $\varepsilon=0.007$ and $\Delta t=0.000005$. Now, we consider the case when $\varepsilon=0.007$ and $\Delta t=0.000005$. In this case, we observe that

$$
\begin{aligned}
\frac{\Delta t}{(\Delta x)^{2}} & =\frac{0.000005}{(0.005)^{2}}=0.2=\frac{1-c_{0}}{2} \\
\frac{c_{0} \delta \varepsilon^{2}}{1-\delta} & =\frac{0.6 \times 0.01 \times(0.007)^{2}}{1-0.01} \\
& =0.00000029696969 \ldots
\end{aligned}
$$

Therefore, we have

$$
\frac{c_{0} \delta \varepsilon^{2}}{1-\delta}=0.00000029696969 \cdots<\Delta t,
$$




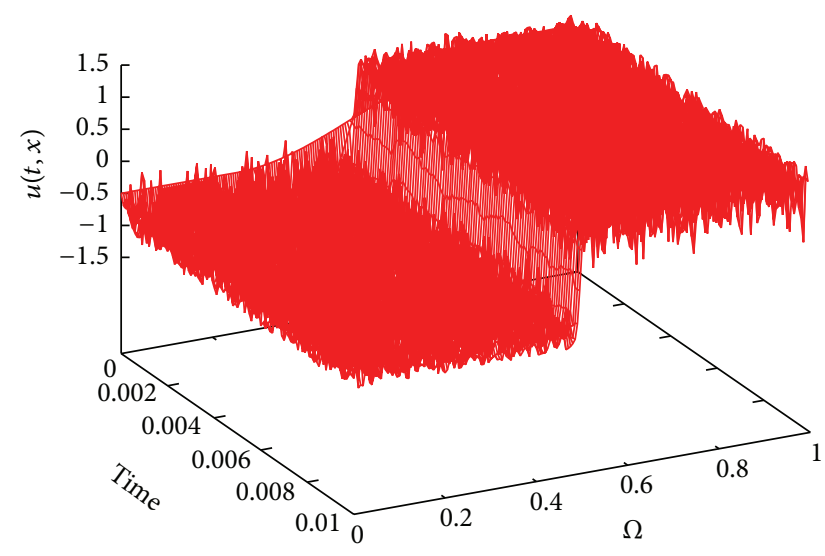

FIGURE 10: $\varepsilon=0.007, \Delta t=0.000005, \Delta x=0.005$, and $\delta=0.01$.

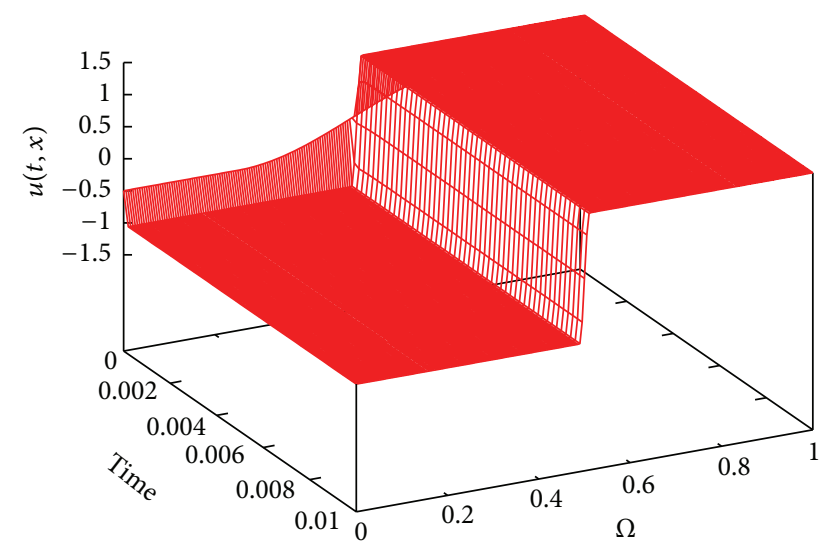

FIGURE 11: $\varepsilon=0.007, \Delta t=0.0000002, \Delta x=0.005$, and $\delta=0.01$.

which implies that criteria condition (63) does not hold. Therefore, we provide the unstable numerical experiment of $(D P)_{\delta}^{\varepsilon}$. Indeed, we have Figure 10.

5.3. The Case When $\varepsilon=0.007$ and $\Delta t=0.0000002$. Now, we consider the case when $\varepsilon=0.007$ and $\Delta t=0.0000002$. In this case, we have

$$
\begin{aligned}
& \frac{\Delta t}{(\Delta x)^{2}}=\frac{0.0000002}{(0.005)^{2}}=\frac{1}{125} \leq 0.2=\frac{1-c_{0}}{2}, \\
& \frac{c_{0} \delta \varepsilon^{2}}{1-\delta}=0.00000029696969 \cdots>\Delta t,
\end{aligned}
$$

which implies that criteria condition (63) holds. Therefore, we provide the stable numerical experiment of $(D P)_{\delta}^{\varepsilon}$. Indeed, we have Figure 11, which implies the stable numerical experiment of $(D P)_{\delta}^{\varepsilon}$.

Remark 19. We observe from Theorem 16 that, in order to get the stable numerical results of $(D P)_{\delta}^{\varepsilon}$, we have to choose the suitable constants $\varepsilon$ and $\delta$ and the mesh size of time $\Delta t$ and space $\Delta x$. Therefore, if we perform a numerical experiment of $(P)^{\varepsilon}$ for sufficient small $\varepsilon$, we had better consider the original problem $(P)^{\varepsilon}$ by using a primal-dual active set method in [2], a Lagrange multiplier method in [9], and so on.
5.4. Conclusion of PDE Problem $(D P)_{\delta}^{\varepsilon}$. By Theorem 16 and the numerical experiments as above, we conclude that the mesh size of time $\Delta t$ and space $\Delta x$ must be satisfied:

$$
\begin{aligned}
& 0<\Delta t \leq \frac{c_{0} \delta \varepsilon^{2}}{1-\delta}, \\
& 0 \leq \frac{\Delta t}{(\Delta x)^{2}} \leq \frac{1-c_{0}}{2}
\end{aligned}
$$

$$
\text { for some constant } c_{0} \in(0,1) \text {, }
$$

in order to get the stable numerical experiments of $(D P)_{\delta}^{\varepsilon}$. Also, by Theorems 7 and 16, we conclude that the value $\delta \varepsilon^{2} /(1-\delta)$ is very important to perform a numerical experiment of $(D E)_{\delta}^{\varepsilon}$ and $(D P)_{\delta}^{\varepsilon}$.

\section{Competing Interests}

The authors declare that there are no competing interests regarding the publication of this paper.

\section{Acknowledgments}

This work was supported by Grant-in-Aid for Scientific Research (C) no. 26400179, JSPS.

\section{References}

[1] S. M. Allen and J. W. Cahn, "A microscopic theory for antiphase boundary motion and its application to antiphase domain coarsening," Acta Metallurgica, vol. 27, no. 6, pp. 1085-1095, 1979.

[2] L. Blank, H. Garcke, L. Sarbu, and V. Styles, "Primal-dual active set methods for Allen-Cahn variational inequalities with nonlocal constraints," Numerical Methods for Partial Differential Equations, vol. 29, no. 3, pp. 999-1030, 2013.

[3] L. Bronsard and R. V. Kohn, "Motion by mean curvature as the singular limit of Ginzburg-Landau dynamics," Journal of Differential Equations, vol. 90, no. 2, pp. 211-237, 1991.

[4] X. Chen, "Generation and propagation of interfaces for reaction-diffusion equations," Journal of Differential Equations, vol. 96, no. 1, pp. 116-141, 1992.

[5] X. Chen and C. M. Elliott, "Asymptotics for a parabolic double obstacle problem," Proceedings of the Royal Society. London. Series A. Mathematical, Physical and Engineering Sciences, vol. 444, no. 1922, pp. 429-445, 1994.

[6] P. de Mottoni and M. Schatzman, "Evolution géométrique d'interfaces," Comptes Rendus de l'Académie des Sciences. Série 1, Mathématique, vol. 309, no. 7, pp. 453-458, 1989.

[7] P. C. Fife, Dynamics of Internal Layers and Diffusive Interfaces, vol. 53 of CBMS-NSF Regional Conference Series in Applied Mathematics, Society for Industrial and Applied Mathematics (SIAM), Philadelphia, Pa, USA, 1988.

[8] M. H. Farshbaf-Shaker, T. Fukao, and N. Yamazaki, "Singular limit of Allen-Cahn equation with constraints and its Lagrange multiplier," in Dynamical Systems, Differential Equations and Applications, AIMS Proceedings, pp. 418-427, AIMS, 2015.

[9] M. H. Farshbaf-Shaker, T. Fukao, and N. Yamazaki, "Lagrange multiplier and singular limit of double-obstacle problems for 
the Allen-Cahn equation with constraint," Mathematical Methods in the Applied Sciences, 2016.

[10] A. Ito, "Asymptotic stability of Allen-Cahn model for nonlinear Laplacian with constraints," Advances in Mathematical Sciences and Applications, vol. 9, no. 1, pp. 137-161, 1999.

[11] N. Kenmochi and K. Shirakawa, "Stability for a parabolic variational inequality associated with total variation functional," Funkcialaj Ekvacioj. Serio Internacia, vol. 44, no. 1, pp. 119-137, 2001.

[12] T. Ohtsuka, "Motion of interfaces by an Allen-Cahn type equation with multiple-well potentials," Asymptotic Analysis, vol. 56, no. 2, pp. 87-123, 2008.

[13] T. Ohtsuka, K. Shirakawa, and N. Yamazaki, "Optimal control of a singular diffusion equation with constraint," Advances in Mathematical Sciences and Applications, vol. 18, no. 1, pp. 1-28, 2008.

[14] T. Ohtsuka, K. Shirakawa, and N. Yamazaki, "Optimal control problem for Allen-Cahn type equation associated with total variation energy," Discrete and Continuous Dynamical Systems, Series S, vol. 5, no. 1, pp. 159-181, 2012.

[15] K. Shirakawa and M. Kimura, "Stability analysis for AllenCahn type equation associated with the total variation energy," Nonlinear Analysis. Theory, Methods \& Applications, vol. 60, no. 2, pp. 257-282, 2005.

[16] Y. Tonegawa, "Integrality of varifolds in the singular limit of reaction-diffusion equations," Hiroshima Mathematical Journal, vol. 33, no. 3, pp. 323-341, 2003.

[17] X. Feng and A. Prohl, "Numerical analysis of the AllenCahn equation and approximation for mean curvature flows," Numerische Mathematik, vol. 94, no. 1, pp. 33-65, 2003.

[18] X. Feng, H. Song, T. Tang, and J. Yang, "Nonlinear stability of the implicit-explicit methods for the Allen-Cahn equation," Inverse Problems and Imaging, vol. 7, no. 3, pp. 679-695, 2013.

[19] J. Shen and X. Yang, "Numerical approximations of AllenCAHn and CAHn-Hilliard equations," Discrete and Continuous Dynamical Systems. Series A, vol. 28, no. 4, pp. 1669-1691, 2010.

[20] X. Yang, "Error analysis of stabilized semi-implicit method of Allen-Cahn equation," Discrete and Continuous Dynamical Systems Series B, vol. 11, no. 4, pp. 1057-1070, 2009.

[21] J. Zhang and Q. Du, "Numerical studies of discrete approximations to the Allen-Cahn equation in the sharp interface limit," SIAM Journal on Scientific Computing, vol. 31, no. 4, pp. 30423063, 2009.

[22] H. Brézis, Opérateurs Maximaux Monotones et Semi-Groupes de Contractions dans les Espaces de Hilbert, North-Holland, Amsterdam, The Netherlands, 1973.

[23] H. Brezis, M. G. Crandall, and A. Pazy, "Perturbations of nonlinear maximal monotone sets in Banach space," Communications on Pure and Applied Mathematics, vol. 23, pp. 123-144, 1970.

[24] A. Ito, N. Yamazaki, and N. Kenmochi, "Attractors of nonlinear evolution systems generated by time-dependent subdifferentials in Hilbert spaces," in Dynamical Systems and Differential Equations, vol. 1 of Discrete and Continuous Dynamical Systems, pp. 327-349, American Institute of Mathematical Sciences, Springfield, Mo, USA, 1996.

[25] M. Ôtani, "Nonmonotone perturbations for nonlinear parabolic equations associated with subdifferential operators, Cauchy problems," Journal of Differential Equations, vol. 46, no. 2, pp. 268-299, 1982.
[26] U. Mosco, "Convergence of convex sets and of solutions of variational inequalities," Advances in Mathematics, vol. 3, pp. 510-585, 1969.

[27] H. Attouch, Variational Convergence for Functions and Operators, Pitman Advanced Publishing Program, Boston, Mass, USA, 1984.

[28] N. Kenmochi, "Monotonicity and compactness methods for nonlinear variational in-equalities," in Handbook of Differential Equations, Stationary Partial Differential Equations, M. Chipot, Ed., vol. 4, chapter 4, pp. 203-298, North-Holland Publishing, Amsterdam, The Netherlands, 2007. 


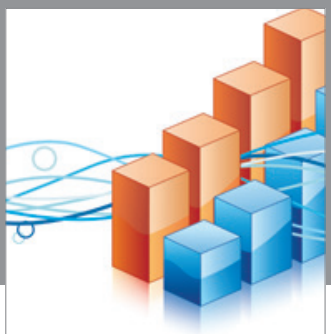

Advances in

Operations Research

vatem alat4

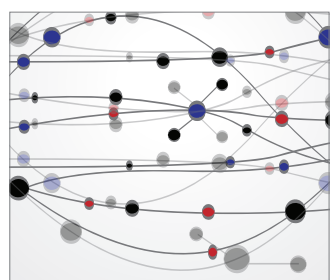

\section{The Scientific} World Journal
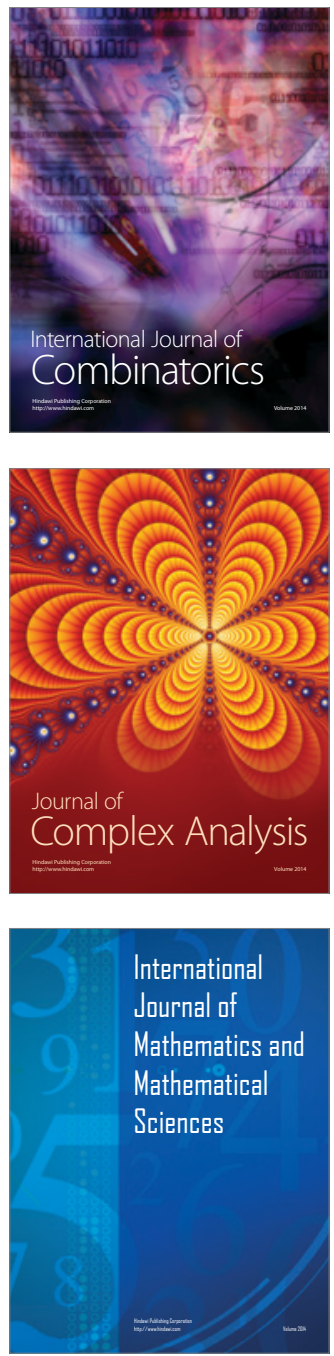
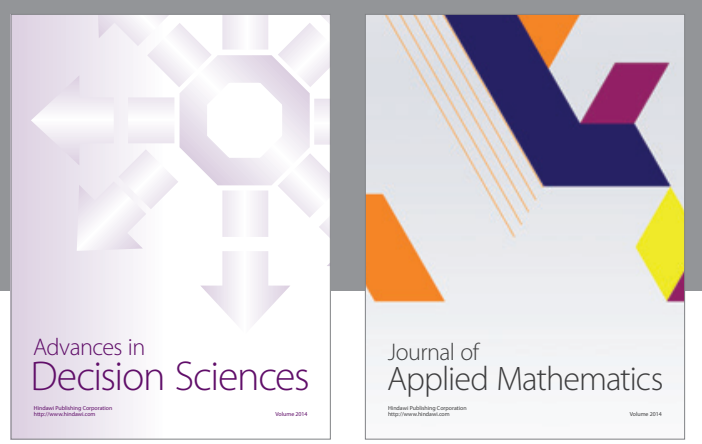

Algebra

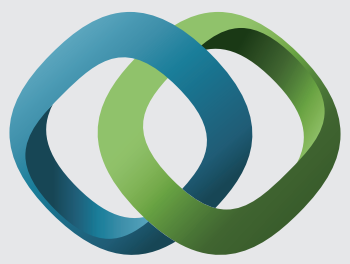

\section{Hindawi}

Submit your manuscripts at

http://www.hindawi.com
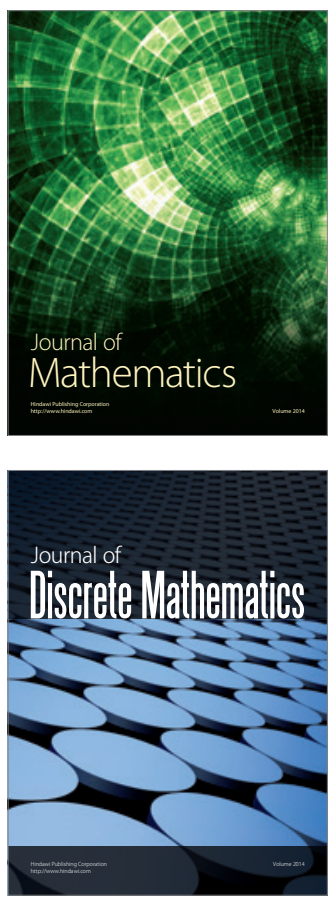

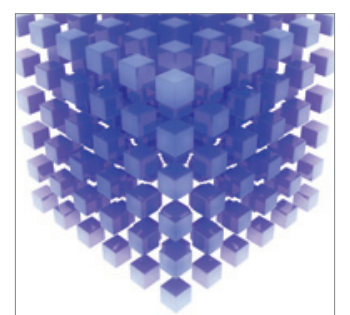

Mathematical Problems in Engineering
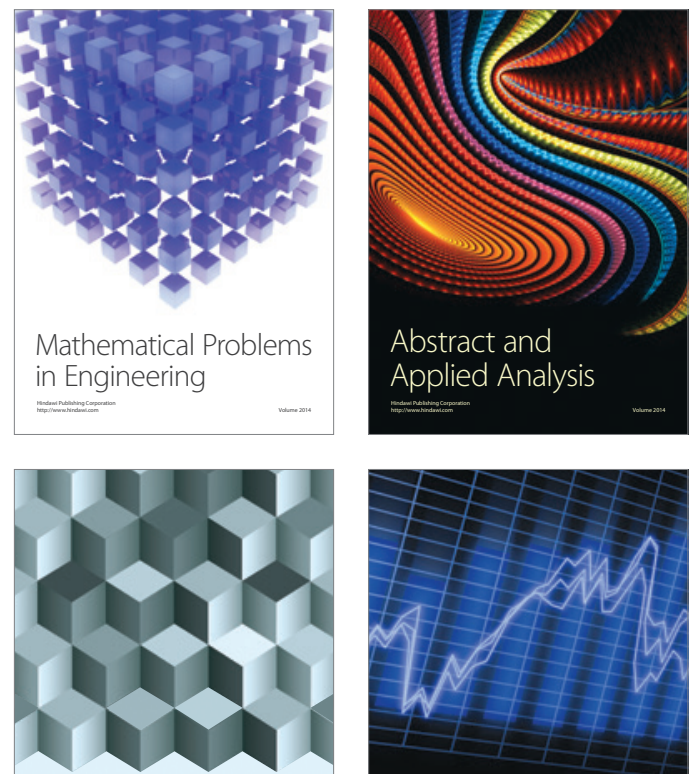

Journal of

Function Spaces

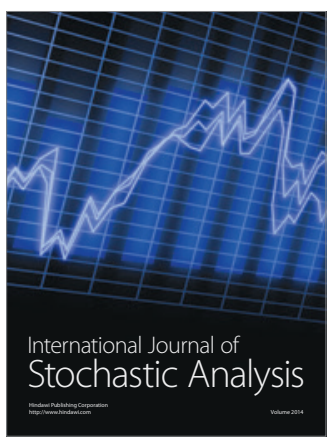

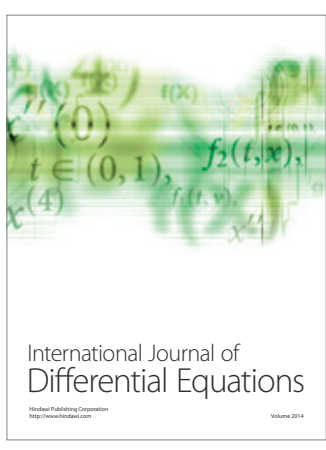
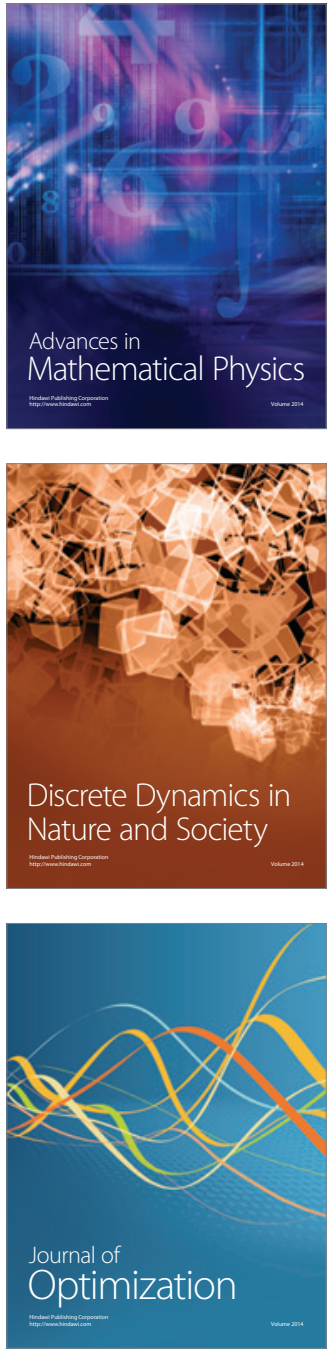